\title{
Cronin Effect in Drell-Yan Reaction
}

\author{
M.B. Johnson ${ }^{1}$, B.Z. Kopeliovich ${ }^{2,3}$ and Ivan Schmidt ${ }^{2}$ \\ ${ }^{1}$ Los Alamos National Laboratory, Los Alamos, NM 87545, USA \\ ${ }^{2}$ Departamento de Física, Universidad Técnica Federico Santa María, \\ Casilla 110-V, Valparaíso, Chile \\ ${ }^{3}$ Joint Institute for Nuclear Research, Dubna, 141980 Moscow Region, Russia
}

\begin{abstract}
We explore the mechanism of transverse momentum broadening of fast quarks propagating in nuclei, using Drell-Yan (DY) transverse momentum distributions measured in the experiment E866 at FermiLab with beams of $800 \mathrm{GeV}$ protons. Our theoretical analysis is based on the color dipole approach in the target rest frame, which has provided a successful phenomenological description of a variety of hadronic reactions. The present application is relevant to the regime of short coherence length (SCL), where the spatial extent of the fluctuations of the projectile responsible for the Drell-Yan reaction is short compared to the internucleon spacing. In this limit, momentum broadening comes from initial state interactions and is described as color filtering, i.e. absorption of large-size dipoles leading to diminished transverse separation and hence enhanced transverse momentum. The predictions we present are in good agreement with the E866 data. The interactions leading to the acquisition of transverse momentum arise from the color-dipole cross section determined previously from deep-inelastic scattering on proton targets. Aside from the determination of the color-dipole cross section, no other phenomenological input is needed to explain the experimental results. The mean-square momentum broadening of dileptons determined in a recent separate analysis of the data is likewise well described by our theory. These results confirm within the model studied that the origin of momentum broadening in DY is the color dipole cross section mediating soft initial state interactions between the parton of the projectile that initiates the reaction and the nucleons of the nucleus, as provided by the color dipole description. Predictions for broadening observables at RHIC are presented.
\end{abstract}




\section{Introduction}

Experimental facilities capable of studying collisions of heavy nuclei at ultra relativistic energies, such as the Relativestic Heavy Ion Collider (RHIC) at BNL and the future Large Hadron Collider (LHC) at CERN, have been motivated by the expectation that data from such collisions will reveal new properties of matter under extreme conditions of temperature and density. In recent years, interpretations of data indeed suggest that during the early stages of these collisions temperatures and densities are created capable of supporting the quark-gluon plasma (QGP), a state of matter never before produced in the laboratory and one of the more dramatic predictions of quantum chromodynamics (QCD), the theory of the strong interactions. For this reason, there is great interest in these experiments and in the challenging theoretical problem of determining properties of the equation of state from the data. These challenges derive in part from the difficulties in unfolding the equation of state from uncertainties in describing the reaction itself. To help resolve these difficulties, simpler nucleon-nucleus and deuteron-nucleus experiments, where the QGP is not produced, have been performed under conditions of energy and momentum transfer similar to those encountered in the nucleus-nucleus collisions.

Some of the most important issues that can be resolved in nucleon-nucleus reactions are related to parton propagation, for example, deciding how quarks and gluons loose energy and acquire transverse momentum as they traverse nuclei under various conditions of coherence. The issues of parton propagation can be cleanly studied in Drell-Yan (DY) reactions in which lepton pairs produced with hadronic beams are detected under controlled kinematic conditions. The important characteristic controlling nuclear effects in the DY reaction is the coherence length, or equivalently the coherence time, which is the lifetime of the hard fluctuation containing the lepton pair. In the regime of short coherence length (SCL), which is of particular interest in this paper, the hard reaction occurs inside the nucleus over a short time scale, thus enabling one to identify the stage of initial state interactions, i.e. those preceding the hard interaction. Thus, the DY reaction in the SCL is clean in the sense that the produced lepton pair hardly interacts at all on its way out of the nucleus, conveying undisturbed information about how the parton of the projectile that initiates the reaction propagates in the nucleus before the production of the lepton pair occurs. The coherence length increases with energy, and in the regime of long coherence length (LCL) one can no longer separate the stages of initial and final state interactions. In this case the whole nucleus acts as a single scattering center.

Notice that only in the LCL regime one can rely on QCD factorization and translate multiple interactions of the projectile partons in the nuclear target into a modification of the nuclear parton distribution function (PDF). In the SCL regime the initial state interactions are known [1] to break factorization.

To be useful, it is necessary to have good statistics under the appropriate kinematical conditions. FermiLab data in $p+A$ collisions [2-5] are useful in these regards and have been analyzed [5-8] as a source of information about both quark energy loss and transverse momentum broadening in nuclei.

In this paper we are particularly interested in momentum broadening of quarks prop- 
agating in nuclei. Theoretical frameworks that have been used to examine momentum broadening in various contexts include the parton model [9-11], perturbative QCD factorization [12], diagrammatic multiple scattering [13], and the color-dipole approach [14-17]. All these theories depend on a phenomenological characterization of the strong interaction to account for non perturbative physics that at present can not be calculated directly from QCD. Accordingly, most require a many-body transport coefficient that is fitted to nuclear scattering data. An exception is the color-dipole approach, where the non perturbative input is fixed at the two-body level by deep-inelastic scattering on protons.

Fixing the the non perturbative input at the two-body level is advantageous when the theoretical formulation is able to use this information as the basic building block for predictions of projectile-nucleus scattering. This is precisely what is done in the color-dipole approach, where nuclear reactions are calculated, using Glauber-Gribov theory [18, 19] and its generalizations [20], in terms of a color-dipole cross section fit to the deep inelastic data. Consequently, once confirmed under more highly controlled experimental conditions, the color-dipole theory can be applied with a high level of confidence in more complicated situations where the transport coefficients may not be able to be determined because the required nuclear data is unavailable. Other desirable features of the color-dipole approach include the fact that since it does not entail a twist expansion, all higher twist terms are naturally incorporated. Another is that it does not need to be corrected for next-to-leading order effects since gluon radiation is already included in the dipole cross section.

The most important advantage of the color-dipole approach is that nuclear effects are predicted rather than fitted to data. In this work we will implement its description of momentum broadening as developed in Ref. [15], and compare its predictions to the DY data mentioned above, with specific interest in refining our understanding of how partons propagate in nuclei. The data consist of ratios of differential transverse momentum distributions $\sigma_{D Y}^{p A}\left(p_{T}\right)$ for a variety of nuclei $[2-5]$

$$
R^{A / A^{\prime}}\left(p_{T}\right)=\frac{\sigma_{D Y}^{p A}\left(p_{T}\right)}{\sigma_{D Y}^{p A^{\prime}}\left(p_{T}\right)},
$$

where $p_{T}$ is the transverse momentum of the detected DY dilepton pair.

The $A$-dependence of the mean-square transverse momentum $\Delta\left\langle p_{T}^{2}\right\rangle$

$$
\Delta\left\langle p_{T}^{2}\right\rangle=\left\langle p_{T}^{2}\right\rangle^{A}-\left\langle p_{T}^{2}\right\rangle^{N}
$$

of the dilepton pair has been of special interest in previous studies of parton propagation. Extraction of the $A$-dependence of $\Delta\left\langle p_{T}^{2}\right\rangle$ was attempted in Ref. [7], using the momentum distribution ratios from the E772 experiment [2]. Unfortunately, $\Delta\left\langle p_{T}^{2}\right\rangle$ can not be uniquely determined from the ratios, requiring instead the individual cross sections appearing in Eq. (1). A number of puzzles have arisen from the results of this analysis, including an apparent inconsistency with theoretical expectations [8,21]. The situation remains unresolved, but a reanalysis [8] of the E772 data along with more recent E866 data [5, 22] shows that the experimental errors on $\Delta\left\langle p_{T}^{2}\right\rangle$ were underestimated in the original analysis, suggesting that there may in fact be no contradiction between theory and experiment. 
Because of this impasse, it is particularly interesting to ask whether the theory describing these momentum distribution ratios agrees with the phenomenological value of $\Delta\left\langle p_{T}^{2}\right\rangle$ that has been determined from them. Thus, an additional goal of our work is to determine the $\Delta\left\langle p_{T}^{2}\right\rangle$ corresponding to the momentum distributions predicted in the color-dipole approach and to compare them to the recent phenomenological analysis given in Ref. [8].

Our paper is organized as follows. In Sect. 2 we introduce the color-dipole model applied to the transverse momentum distributions for the DY reaction on individual hadrons. Section 3 discusses the extension of the theory to DY reactions on nuclei and the way in which the nuclear medium modifies these distributions. Section 3.1 addresses the important issue of coherence length, and Sects. 3.2 presents the theory and its implementation as applied to DY on nuclei. Section 3.3 gives numerical results for the ratios of momentum distributions in comparison to experiment, and Sect. 3.4 gives the corresponding results for $\Delta\left\langle p_{T}^{2}\right\rangle$. Finally, in Sect. 3.5, we present predictions for RHIC. Various technical details justifying the approximations made when evaluating the theory are relegated to the Appendix. Section 4 summarizes the paper and presents our conclusions.

\section{Drell-Yan reaction on a nucleon in the target rest frame}

The color dipole approach in the target rest frame provides an attractive means for interpreting DY data, for reasons discussed in the Introduction. The color dipole approach was originally proposed in [23] for hadronic interactions, and it was applied to deep-inelastic scattering (DIS) in [24]. Extension to Drell-Yan was developed by Kopeliovich [25] and subsequently by Brodsky et al. [26]. According to this picture, the Drell-Yan reaction occurs in the target rest frame when a fast projectile quark scatters off the gluonic field of the target, radiating a massive photon $\gamma^{*}$ of mass $M$ as shown in Fig. 1. In this frame, the $\gamma^{*}$ is a constituent of the projectile fluctuations, which are "frozen" by time dilation for a length of time $t_{c}$, the coherence time, given by the uncertainty relation,

$$
t_{c}=\frac{2 E_{q}}{M_{q \bar{l} l}^{2}-m_{q}^{2}},
$$

where $E_{q}$ and $m_{q}$ refer to the energy and mass of the projectile quark and $M_{q \bar{l} l}^{2}$ is the square of the effective mass of the fluctuation,

$$
M_{q \bar{l} l}^{2}=\frac{m_{q}^{2}}{\alpha}+\frac{M_{\overline{l l}}^{2}}{1-\alpha}+\frac{k_{T}^{2}}{\alpha(1-\alpha)},
$$

with $\alpha$ the fraction of the light-cone momentum of the incident quark carried by the lepton pair and $k_{T}$ the transverse momentum of the lepton pair. The DY reaction occurs when the $\gamma^{*}$ of the fluctuation is released by an interaction between one of the constituents of the

fluctuation and a target nucleon. Subsequently the $\gamma^{*}$ decays into the observed Drell-Yan dilepton pair $\bar{l}$. 

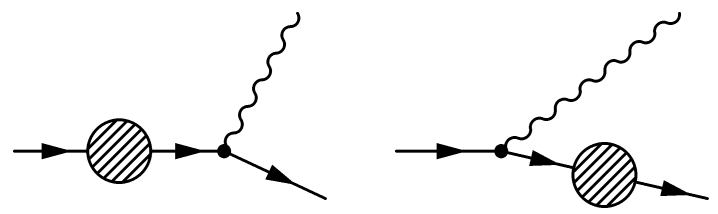

Figure 1: In the target rest frame, DY dilepton production looks like bremsstrahlung. A quark or an anti-quark from the projectile hadron scatters off the target color field (denoted by the shaded circles) and radiates a massive photon, which decays into the lepton pair. The photon decay is not shown. The photon can be radiated before or after the quark scatters.

The DY cross section on a nucleon is described by

$$
M^{2} \frac{d^{2} \sigma_{D Y}}{d M^{2} d x_{1}}=\frac{\alpha_{e m}}{6 \pi x_{1}} \int_{x_{1}}^{1} d x_{q} F_{q}^{h}\left(x_{q}\right) \int d^{2} \rho|\Psi(\alpha, \rho)|^{2} \sigma_{\bar{q} q}(\alpha \rho),
$$

where $\sigma_{\bar{q} q}(r)$ is the universal color-dipole cross section, $\rho$ is the transverse distance between the $\gamma^{*}$ and quark in the fluctuation, and $\Psi$ represents the light-cone distribution amplitude for the incident quark to fluctuate into a quark and the $\gamma^{*}$. In DY, the color dipole consists of the quark before and after the release of the $\gamma^{*}$, whose impact parameters differ by $\alpha \rho$. In Eq. (5) $x_{q}\left(x_{1}\right)$ is the fraction of light-cone momentum of the incoming hadron $h$, for us a proton, carried by the quark (lepton pair) and $F_{q}^{h}$ is the average quark distribution function of the incident proton,

$$
F_{q}^{h}\left(x_{q}\right)=\sum_{f}\left[q_{f}\left(x_{q}\right)+q_{\bar{f}}\left(x_{q}\right)\right] .
$$

The light-cone momentum fraction $\alpha$ is given in terms of these variables as $\alpha=x_{1} / x_{q}$.

Deep-inelastic scattering from nucleons at HERA (DESY) has been used to fix models of the color-dipole cross section. The HERA data suggests that the color dipole cross section saturates at large $r_{T}$, i.e. approaches a constant value. Golec-Biernat and Wüsthoff (GW) chose a simple saturated form for the color dipole cross section [27] and found that the following expression,

$$
\sigma_{\bar{q} q}\left(r_{T}, x\right)=\sigma_{0}\left(1-e^{-r_{T}^{2} / R_{0}^{2}(x)}\right),
$$


parametrizes well these data. For small $r_{T}$,

$$
\sigma_{\bar{q} q}\left(r_{T}\right) \approx C r_{T}^{2}
$$

where the factor $C=\sigma_{0} / R_{0}^{2}(x)$ depends on Bjorken $x$. For us, the most important point is that once the color dipole cross section has been fitted to experimental DIS data on the nucleon, the color-dipole formalism makes predictions for Drell-Yan both in nucleonnucleon [28] and nucleon-nucleus scattering with no further adjustment of parameters.

The GW theory is known to describe DY on a nucleon, in the color dipole approach, only at small Bjorken $x$, and specific calculations [28] for $x_{2}<0.1$ have shown it to agree well both in the magnitude and shape with the next-to-leading order parton model. Although the calculations disagreed with the E772 FermiLab data, they are in excellent agreement with data from the E866 experiment that has recently become available [22].

An expression similar to Eq. (5) exists for the momentum distribution.

$$
\frac{d^{4} \sigma_{D Y}}{d M^{2} d x_{F} d^{2} p_{T}}=\frac{\alpha_{e m}}{3 \pi M^{2}} \frac{x_{1}}{x_{1}+x_{2}} \int_{x_{1}}^{1} \frac{d \alpha}{\alpha^{2}} F_{q}^{h}\left(\frac{x_{1}}{\alpha}\right) \frac{d \sigma\left(q N \rightarrow \gamma^{*} X\right)}{d \ln \alpha d^{2} p_{T}} .
$$

The differential cross section for heavy-photon radiation in a quark-nucleon collision was derived in [30],

$$
\begin{aligned}
\frac{d \sigma\left(q N \rightarrow \gamma^{*} X\right)}{d \ln \alpha d^{2} k_{T}} & =\frac{1}{(2 \pi)^{2}} \int d^{2} \rho_{1} d^{2} \rho_{2} \exp \left[i \vec{k}_{T} \cdot\left(\vec{\rho}_{1}-\vec{\rho}_{2}\right)\right] \Psi_{\gamma^{*} q}^{*}\left(\alpha, \vec{\rho}_{1}\right) \Psi_{\gamma^{*} q}\left(\alpha, \vec{\rho}_{2}\right) \\
& \times \frac{1}{2}\left[\sigma_{q \bar{q}}\left(\alpha \rho_{1}\right)+\sigma_{q \bar{q}}\left(\alpha \rho_{2}\right)-\sigma_{q \bar{q}}\left(\alpha\left|\vec{\rho}_{1}-\vec{\rho}_{2}\right|\right)\right] .
\end{aligned}
$$

This is described in detail in Ref. [29]. In this case, the transverse momentum $p_{T}$ of the DY pair is measured. The DY variables $x_{1}, M^{2}, x_{2}$, and $k_{T}$ satisfy the equations,

$$
\begin{gathered}
x_{1} x_{2}=\frac{M^{2}+k_{T}^{2}}{s}, \\
x_{1}-x_{2}=x_{F},
\end{gathered}
$$

where $x_{F}$ is the Feynman variable. The variables $x_{1}$ and $x_{2}$ are interpreted in the parton model as the Bjorken variables of the annihilating quark and antiquark.

Since the virtual photon of Eq. (10) originates in a projectile fluctuations at the hard scale, the quark distribution function over which this cross section is averaged to get the Drell-Yan reaction should be taken at the same scale. Additionally, in principle the distribution function should be of leading order since gluon radiations are included in the dipole cross section. In practice, the cross section ratios in which we are interested are rather sensitive to the details of the choice of the quark distribution function, and for our calculations we use a simple phenomenological choice [31]. 


\section{Medium effects in DY reactions in the target rest frame}

When the scattering takes place on a nucleus, multiple interactions with target nucleons can give rise to various medium effects. The coherence length determines how these arise, and it is useful to distinguish two limiting cases in which the theory simplifes. One is the SCL limit, reached when the coherence length $\ell_{c} \equiv c\left\langle t_{c}\right\rangle$ becomes much smaller than the interparticle spacing $d, \ell_{c} \ll d$ (in a heavy nucleus, $d \approx 2 f m$ ). The other is the LCL limit, reached when $\ell_{c} \gg R_{A}$, where $R_{A}$ is the nuclear radius.

One of the important medium effects is shadowing. In the SCL there is no shadowing because the duration of the fluctuation is so short that the constituents of the fluctuation have no time to multiply interact with the medium. In the LCL, which is applicable to reactions at the $\mathrm{LHC}$ and at $\mathrm{RHIC}$ under certain kinematic conditions, there is maximal shadowing.

Interactions of partons in the nuclear medium also give rise to parton energy loss and to transverse momentum broadening, to which ratios of nuclear cross sections $R^{A / A^{\prime}}$ are sensitive. In DY, these effects arise in the SCL as partons from the incident hadron undergo soft interactions with nucleons of the nucleus before experiencing a hard interaction that immediately liberates a $\gamma^{*}$ from one of its short-lived fluctuations. For the LCL, the nuclear interactions that lead to shadowing are also a source of parton momentum broadening. Nuclear effects giving rise to momentum broadening and shadowing are believed to arise predominantly from the same color dipole cross section $\sigma_{\bar{q} q}\left(r_{T}\right)$ that mediates the DY reaction on a nucleon.

Cases intermediate between the SCL and LCL are generally more difficult to model. Several methods have been proposed for this. The most strict one is the Green function method [20]. Another one is a simple interpolation between the SCL and LCL limits using the square of the longitudinal form factor $F_{A}^{2}\left(q_{c}\right)$ expressed in terms of the longitudinal momentum transferred in the reaction, $q_{c}=1 / l_{c}$. The latter method was recently applied to determine quark energy loss from ratios of $p_{T}$-integrated nuclear DY cross sections, using E772/E866 FermiLab data at $E_{p}=800 \mathrm{GeV} 1$. The kinematics of this data corresponds, for the most part, to the SCL regime; therefore, initial state interactions are predominantly soft and they decouple from the hard DY reaction.

Predictions for nuclear broadening in DY reaction based on the theory [30] for the long coherence length limit were recently given in Ref. [29]. Here we concentrate on the SCL limit for which the theory to describe quark transverse momentum broadening has been presented in Ref. [15].

\footnotetext{
${ }^{1}$ In this analysis energy loss was assumed to be independent of the parton energy in accordance with perturbative QCD calculations. However, for soft constituent partons an effective energy loss turns out to rise with energy [32]. Nevertheless, this fact does not affect the results of the analysis [6], but only their applications at higher or lower energies.
} 


\subsection{Coherence length vs transverse momentum}

As discussed in the previous section, there are two important limits of coherence length, those of SCL and of LCL, and distinct mechanisms of transverse momentum broadening apply in each of them. Experimental access to the LCL regime requires beams of sufficiently high energy to allow fluctuations of the projectile to be frozen by Lorentz time dilation so that their spatial extent exceeds the nuclear diameter. We do not have so far any DY measurements to verify the mechanism of transverse momentum broadening at play in this limit, although predictions for $p+A, d+A$, and $A+A$ collisions at RHIC at $x_{F}=x_{1}-x_{2}>0.5$ and at the LHC are available [29]. The experimental situation is different for the SCL limit. The most extensive data sets we have today are the momentum distributions of the E772/E866 FermiLab experiments. To better define the dividing line between the two regimes for the momentum distributions, we examine in this section the coherence length as a function of transverse momentum.

We proceed by calculating the coherence length of the fluctuation in the incident proton containing the $\gamma *$ of transverse momentum $k_{T}$ that eventually decays into a dilepton pair of momentum $p_{T}$ as measured in the laboratory. We proceed as in Ref [6], averaging Eqs. (3](4) over $\alpha$,

$$
\left\langle l_{c}\right\rangle=\frac{1}{m_{N} x_{2}} \frac{\int_{x_{1}}^{1} d x_{q} F_{q}^{h}\left(x_{q}\right) \int d^{2} k_{T}\left|\widetilde{\Psi}_{\bar{l} l q}\left(x_{1} / x_{q}, k_{T}\right)\right|^{2} K^{D Y}\left(x_{1} / x_{q}\right)}{\int_{x_{1}}^{1} d x_{q} F_{q}^{h}\left(x_{q}\right) \int d^{2} k_{T}\left|\widetilde{\Psi}_{\bar{l} l q}\left(x_{1} / x_{q}, k_{T}\right)\right|^{2}},
$$

where $K^{D Y}(\alpha)$ is

$$
K^{D Y}(\alpha)=\frac{M^{2}\left(x_{1}, x_{2}, k_{T}\right)(1-\alpha)}{M^{2}\left(x_{1}, x_{2}, k_{T}\right)(1-\alpha)+\alpha^{2} m_{q}^{2}+k_{T}^{2}} .
$$

with

$$
M^{2}\left(x_{1}, x_{2}, k_{T}\right)=s x_{1} x_{2}-k_{T}^{2}
$$

in accord with Eq. (11).

The normalization integral for the light-cone distribution amplitude is ultra-violet divergent (for radiation of transverse photons), implying that the vacuum fluctuations are overwhelmed by infinitely heavy $\left|q \gamma^{*}\right\rangle$ fluctuations. Those heavy fluctuations, however, do not contribute to the DY cross section, since they are too small to be resolved and distinguished from a bare quark. Therefore, a proper weight factors in the above averaging procedure must involve the dipole interaction cross section on top of the light-cone distribution amplitude. The Fourier transform of such a modified light-cone distribution amplitude $\widetilde{\Psi} \frac{T}{l l} q$ was evaluated in Ref. [6] as

$$
\widetilde{\Psi}_{\overline{l l q}}^{T}\left(\alpha, k_{T}\right)=2 Z_{q} \sqrt{\alpha_{e m}} C(s) \vec{e} \cdot \vec{k}_{T} \frac{i \alpha^{2} \tau^{2}}{\pi\left(\tau^{2}+k_{T}^{2}\right)^{3}},
$$

where $\hat{e}$ is the polarization of the virtual photon and $\tau^{2}=(1-\alpha) M^{2}\left(x_{1}, x_{2}, k_{t}\right)+\alpha^{2} m_{q}^{2}$. The calculation was done with the simplified dipole cross section $\sigma_{\bar{q} q}\left(r_{T}, x\right)=C(x) r_{T}^{2}$. 
We show the coherence length for the FermiLab E772/E866 experiment at $s^{1 / 2}=$ $38.3 \mathrm{GeV}$ in Fig. 2. The values of $x_{1}$ and $x_{2}$ relevant to the data for E772 are in the range $0.07<x_{2}<0.13$ and $0.33<x_{1}<0.43$ [35]. The E866 data covers the range $.02<x_{2}<.08$, with $\left\langle x_{1}\right\rangle=0.46$ and $\langle M\rangle=4.6 \mathrm{GeV}$ [36]. The values of $p_{T}$ cover the range from 0 to about $5 \mathrm{GeV} / \mathrm{c}$. Although the coherence length has been evaluated using the value of $x_{1}$

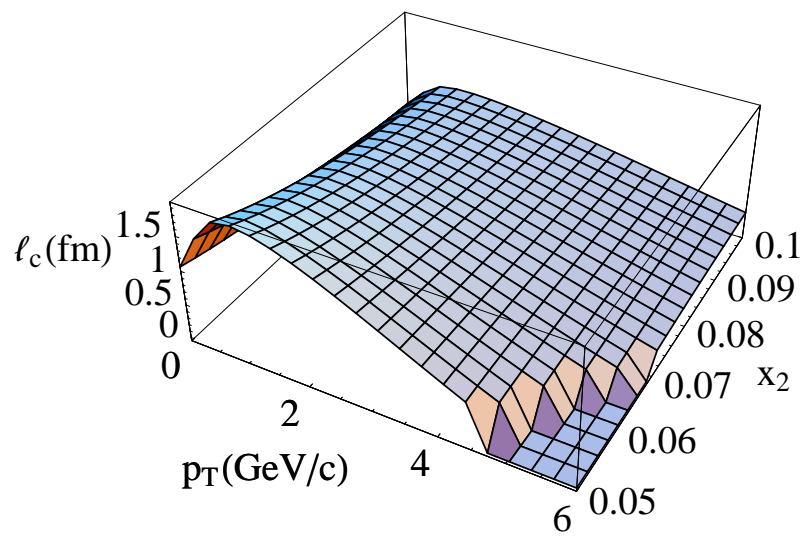

Figure 2: (Color online) Coherence length for FermiLab kinematics.

corresponding to the E866 data, $\left\langle x_{1}\right\rangle=0.46, \ell_{c}$ is rather insensitive to its precise value. We see that the short-coherence length limit $\left(\ell_{c} \approx 2 \mathrm{fm}\right)$ prevails for $x_{2}>0.05$ and for all values of $p_{T}$ beyond $4.5 \mathrm{GeV} / c$. The figure is cut off when $M$ drops below $4 \mathrm{GeV}$. We see that this happens for $p_{T}>4.5 \mathrm{GeV}$ at $x_{2}=0.05$, but for $x_{2}>0.07 M<4 \mathrm{GeV}$ throughout the range of $p_{T}$ shown.

We find the results for RHIC kinematics shown in Fig. 3. This corresponds to $x_{1}=x_{2}$ $\left(x_{F}=0\right)$ and $s^{1 / 2}=200 \mathrm{GeV}$. We see that the coherence length is less than the internuclear spacing for $x_{2}>0.05$ and for all values of $p_{T}$ beyond $9 \mathrm{GeV} / c$.

\subsection{Calculating momentum distribution in the SCL limit}

Calculations of the previous section show that DY production for $x_{2}>0.05$ at FermiLab in the E772/E866 experiments with $800 \mathrm{GeV}$ protons and at RHIC with $s^{1 / 2}=200 \mathrm{GeV}$ protons at $x_{F}=0$ explore momentum distributions in the SCL limit. In this subsection we calculate these distributions using the theory of Ref. [15]. We will compare these calculations to the E866 data in the next subsection. We do not show comparisons with the E772 data because the transverse momentum distributions are essentially identical to those with the more recent and reliable data from E866. Later on, we show predictions for transverse momentum observables under kinematic conditions appropriate to RHIC. 


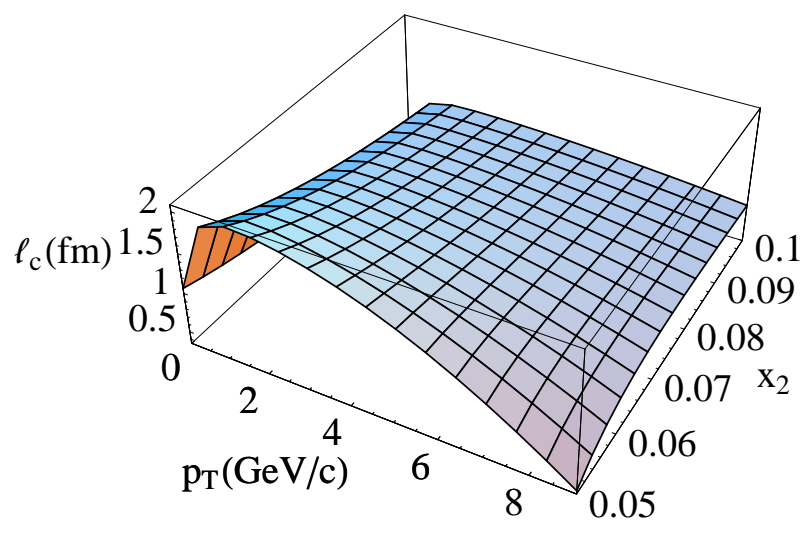

Figure 3: (Color online) Coherence length for RHIC kinematics.

It was recognized in that work that at high energies, the multiple interactions of a quark propagating in the nuclear medium can be eikonalized and exponentiate into a factor containing the color dipole cross section and the nuclear thickness function. The physics of momentum broadening of the quark is therefore that of color filtering, or absorption of large dipoles leading to diminished transverse separation with distance. Then, the probability distribution $W^{q}\left(k_{T}, s_{q}\right)=d n_{q} / d^{2} k_{T}$ that a valence quark arriving at a position $(\vec{B}, z)$ in the nucleus $A$ will have acquired transverse momentum $\vec{k}_{T}$ becomes [15]

$$
W^{q}\left(\vec{k}_{T}, s_{q}, \vec{B}, z\right)=\frac{N}{(2 \pi)^{2}} \int d^{2} b \int d^{2} b^{\prime} e^{i \vec{k}_{T} \cdot\left(\vec{b}-\vec{b}^{\prime}\right)} e^{-\frac{b^{2}+b^{2}}{3\left\langle r_{c m}^{2}\right\rangle}} e^{-\frac{1}{2} \sigma_{\bar{q} q}\left(\vec{b}-\vec{b}^{\prime}, s_{q}\right) T_{A}\left(\frac{\vec{b}+\vec{b}^{\prime}}{2}+\vec{B}, z\right)}
$$

where $s_{q}$ is the square of the quark-nucleon energy in their center-of-mass, and

$$
N=\frac{2}{3 \pi\left\langle r_{c m}^{2}\right\rangle}
$$

with $\left\langle r_{c m}^{2}\right\rangle=0.79 \pm 0.03 \mathrm{fm}^{2}$ the mean-square charge radius of the proton [37], and where $T_{A}(b, z)$ is the nuclear thickness function,

$$
T_{A}(b, z)=\int_{-\infty}^{z} d z \rho_{A}(b, z)
$$

with $\rho_{A}(b, z)$ the nuclear density. To obtain the transverse momentum acquired by a quark on the nucleus, $W^{q A}\left(\vec{k}_{T}, s_{q}, z\right)$, Eq. (17) must be averaged over the nuclear density $\rho_{A}(B, z)$, which entails

$$
W^{q A}\left(\vec{k}_{T}, s_{q}, z\right) \equiv\left\langle W^{q}\left(\vec{k}_{T}, s_{q}, \vec{B}, z\right)\right\rangle_{N}=\frac{1}{A} \int d^{2} B \int d z \rho_{A}(B, z) W^{q}\left(\vec{k}_{T}, s_{q}, \vec{B}, z\right) .
$$


Notice that in the regime of SCL the soft initial state interactions and the hard reaction (DY) cross section factorize. The universal dipole cross section, Eq. (7), fitted to low- $x$ data for $F_{2}\left(x, Q^{2}\right)$, can be appropriately applied to the DY cross section on a proton, but not to the soft multiple rescatterings in nuclear matter described via Eq. (17). For evaluating Eq. (17) we therefore use the KST color-dipole cross section, which covers soft and low- $Q^{2}$ data [39]. For such data Bjorken $x$ is not a proper variable, since the smallness of $x$ does not mean high energy if $Q^{2}$ is small. The KST cross section has the same saturated shape as Eq. (77), but with different parameters that depend explicitly on the quark-nucleon centerof-mass energy. For DY with a proton beam, $s_{q}$ is given by $s_{q}=2 x_{1} m_{N} E_{p}$, where $E_{p}$ is the laboratory energy of the protons and $m_{M}$ is their mass. Soft initial state interactions also lead to gluon shadowing, which we take into account by replacing $\sigma_{\bar{q} q} \rightarrow R_{G}(x, A) \sigma_{\bar{q} q}$, where $R_{G}(x, A)$ is the gluon shadowing function calculated [20] in the Green function approach from the KST model. See Ref. [15] for more discussion. We stress that our theoretical results do not rely on any adjustable parameters.

We express the cross section, $\sigma_{D Y}^{q A}\left(\alpha, p_{T}\right)$, for an incident quark to produce a DY pair on a nucleus $A$ with transverse momentum $p_{T}$ as the convolution of the probability $W^{q A}\left(k_{T}, s_{q}\right)$ with the momentum distribution for a quark to produce a Drell-Yan pair on a proton, $\sigma_{D Y}^{q p}\left(\alpha, k_{T}\right)$

$$
\sigma_{D Y}^{q A}\left(\alpha, p_{T}\right)=\int d^{2} k_{T} W^{q A}\left(k_{T}, s_{q}\right) \sigma_{D Y}^{q p}\left(\alpha, \vec{p}_{T}-\alpha \vec{k}_{T}\right)
$$

To obtain the momentum distribution for an incident proton, this must be averaged over the quark distribution function of the incident proton, as in Eq. (99),

$$
\sigma_{D Y}^{p A}\left(p_{T}\right)=\left\langle\sigma_{D Y}^{q A}\left(\alpha, p_{T}\right)\right\rangle_{\alpha}
$$

where we define the bracket $\langle f(\alpha)\rangle_{\alpha}$ to denote the integral of the function $f(\alpha)$ enclosed in it over the distribution function of a quark in the incident proton,

$$
\langle f(\alpha)\rangle_{\alpha}=\int_{0}^{1} d \alpha d x_{q} F_{q}^{h}\left(x_{q}\right) f(\alpha) \delta\left(\alpha-x_{1} / x_{q}\right)
$$

The combination of momenta $\vec{p}_{T}-\alpha \vec{k}_{T}$ in Eq. (21) accounts for the fact that the DY pair carries away only a fraction $\alpha$ of the transverse momentum $\vec{k}_{T}$ of a quark in the incident proton.

We denote the $\alpha$-dependence of $\sigma_{D Y}^{q p}\left(\alpha, k_{T}\right)$ by $g\left(\alpha, k_{T}\right)$, which we take from Eqs. (9) and (10),

$$
g\left(\alpha, k_{T}\right)=\frac{d \sigma\left(q N \rightarrow \gamma^{*} X\right)}{d \ln \alpha d^{2} k_{T}}\left\langle\frac{d \sigma\left(q N \rightarrow \gamma^{*} X\right)}{d \ln \alpha d^{2} k_{T}}\right\rangle_{\alpha}^{-1}
$$

evaluated using Eqs. (B.10) - (B.13) of Ref. [29]. The transverse momentum distribution $\sigma_{D Y}^{p p}\left(k_{T}\right)$ is of course obtained from $\sigma_{D Y}^{q p}\left(\alpha, k_{T}\right)$ by convolution,

$$
\sigma_{D Y}^{p p}\left(p_{T}\right) \equiv\left\langle\sigma_{D Y}^{q p}\left(\alpha, p_{T}\right)\right\rangle_{\alpha}
$$

as in Eq. (23). Thus,

$$
\sigma_{D Y}^{q p}\left(\alpha, k_{T}\right)=\sigma_{D Y}^{p p}\left(k_{T}\right) g\left(\alpha, k_{T}\right)
$$


and we see that $g\left(\alpha, k_{T}\right)$ is closely related to the probability $P\left(\alpha, k_{T}\right) \equiv g\left(\alpha, k_{T}\right) F_{q}^{h}\left(x_{1} / \alpha\right) / \alpha$ that a quark of the incident proton carrying light cone momentum fraction $\alpha$ of the detected dilepton pair radiates the $\gamma *$ of momentum $k_{T}$ that decays into the pair.

Since the production of the DY pair is a hard reaction, occurring for $M>4 \mathrm{GeV}$, we have used the GW color-dipole cross section to calculate $g\left(\alpha, k_{T}\right)$ in Eq. (24). This said, we note that the only reason we need $g\left(\alpha, k_{T}\right)$ is for the purpose of averaging over $\alpha$, and we therefore expect the transverse momentum distributions on nuclei to be insensitive to the details of the color dipole model used for calculating it.

After performing averages over the nuclear density and integrating over $\alpha$, the momentum distribution of a DY pair produced on a nucleus, $\sigma_{D Y}^{p A}\left(p_{T}\right)$, is given by

$$
\sigma_{D Y}^{p A}\left(p_{T}\right)=\int d^{2} k_{T}\left\langle\left\langle W^{q}\left(k_{T}, s_{q}\right)\right\rangle_{N} \sigma_{D Y}^{q p}\left(\alpha, \vec{p}_{T}-\alpha \vec{k}_{T}\right)\right\rangle_{\alpha}
$$

and the final expression for the nuclear ratio becomes

$$
R^{A / p}\left(p_{T}\right)=\frac{1}{\sigma_{D Y}^{p p}\left(p_{T}\right)} \int d^{2} k_{T}\left\langle\left\langle W^{q}\left(k_{T}, s_{q}\right)\right\rangle_{N} \sigma_{D Y}^{q p}\left(\alpha, \vec{p}_{T}-\alpha \vec{k}_{T}\right)\right\rangle_{\alpha}
$$

Using Eq. (17) in Eq. (21) and changing variables, we may express the transverse momentum distribution of a quark as

$$
\sigma_{D Y}^{q A}\left(\alpha, p_{T}\right)=\frac{1}{A} \int \frac{d^{2} k_{T}}{(2 \pi)^{2}} \int d^{2} r_{T} e^{i k_{T} \cdot r_{T}} e^{-\frac{r_{T}^{2}}{6\left\langle r_{c m}^{2}\right\rangle}} U\left(r_{T}\right) \sigma_{D Y}^{q p}\left(\alpha, \vec{p}_{T}-\alpha \vec{k}_{T}\right)
$$

where

$$
U\left(r_{T}\right)=N \int d^{2} b \int d z \int d^{2} R \rho_{A}(\vec{R}-\vec{b}, z) e^{-\frac{2 R^{2}}{3\left\langle r_{c m}^{2}\right\rangle}} e^{-\frac{1}{2} \sigma_{\bar{q} q}\left(r_{T}, s_{q}\right) T_{A}(b, z)} .
$$

The average over the nucleus is examined in the appendix, where it is shown that to an excellent approximation the effect of averaging Eq. (17) over $(B, z)$ is to replace

$$
T_{A}(b, z) \rightarrow\left\langle T_{A}\right\rangle / 2
$$

which we will refer to as the average thickness function prescription. The average thickness function is defined by

$$
\left\langle T_{A}\right\rangle=\frac{1}{A} \int d^{2} b T(b)^{2} .
$$

We may therefore obtain $\sigma_{D Y}^{p A}\left(p_{T}\right)$ by averaging over $\alpha$ as in Eq. (22) with the average $T_{A}$ prescription, Eq. (31).

In this case, we find a numerically convenient form for $U\left(r_{T}\right)$ by expanding the term $e^{-r_{T}^{2} / R_{0}^{2}}$ of Eq. (7), and thus obtaining,

$$
U\left(r_{T}\right)=A e^{\frac{\sigma_{0}}{2}\left\langle T_{A}\right\rangle / 2} \sum_{L=0} \frac{1}{L !}\left(\frac{\sigma_{0}}{2} \frac{\left\langle T_{A}\right\rangle}{2}\right)^{L} e^{-L r_{T}^{2} / R_{0}^{2}}
$$


This leads to

$$
\sigma_{D Y}^{q A}\left(\alpha, p_{T}\right)=S_{0}(\alpha) \sigma_{D Y}^{q p}\left(\alpha, p_{T}\right)+\int \frac{d^{2} k_{T}}{(2 \pi)^{2}} S_{1}\left(\alpha, \vec{k}_{T}, \vec{p}_{T}\right) \sigma_{D Y}^{q p}\left(\alpha, k_{T}\right)
$$

where

$$
\begin{aligned}
S_{0}(\alpha) & =e^{\frac{\sigma_{0}}{2}\left\langle T_{A}\right\rangle / 2} \\
S_{1}\left(\alpha, k_{T}, p_{T}\right) & =\frac{e^{\frac{\sigma_{0}}{2}\left\langle T_{A}\right\rangle / 2}}{\alpha^{2}} \sum_{L=1} \frac{\left(\frac{\sigma_{0}}{2}\left\langle T_{A}\right\rangle / 2\right)^{L}}{\frac{L}{R_{0}^{2}}+\frac{1}{6\left\langle r_{c m}^{2}\right\rangle}} \frac{\pi}{L !} e^{-\frac{\left(\vec{k}_{T}+\vec{p}_{T}\right)^{2}}{4 \alpha^{2}} \frac{1}{\frac{L}{R_{0}^{2}}+\frac{1}{6\left\langle r_{c m}^{2}\right\rangle}}}
\end{aligned}
$$

as in Eq. (A.13) of the appendix. The $L=0$ term has been pulled out and simplified by noting that the integrand for this term is strongly peaked about $k_{T}=0$ due to the fact that $\left\langle r_{c m}^{2}\right\rangle$ is large on the $\mathrm{GeV}$ scale characterizing $\sigma_{D Y}^{p p}\left(p_{T}\right)$. Notice that in the KST parametrization the parameter $\sigma_{0} \equiv \sigma_{0}(s)$ is energy dependent and thus gets its dependence on $\alpha$ according to the convolution in (21). This dependence, however, has a very little impact on $\left\langle S_{0}\right\rangle_{\alpha}$.

Because $g\left(\alpha, k_{T}\right)$ is strongly peaked around $\alpha=1$, the average $\left\langle S_{1}\left(\alpha, \vec{k}_{T}, \vec{p}_{T}\right)\right\rangle_{\alpha}$ is dominated by the mean value of $\alpha$ contributing to the DY transverse momentum distribution on a nucleon. We call this average $\bar{\alpha}_{1}\left(k_{T}\right)$, defined as

$$
\bar{\alpha}_{1}\left(k_{T}\right) \approx \sqrt{\left\langle\alpha^{2} g\left(\alpha, k_{T}\right)\right\rangle_{\alpha}} .
$$

Physically, $\bar{\alpha}_{1}\left(k_{T}\right)$ is the average light cone momentum fraction of the DY dilepton pair carried by the parton of the incident hadron that radiates the $\gamma *$ of the dilepton pair. Note that this average depends not only on the momentum $k_{T}$ of the $\gamma *$ but also on $x_{1}$. Alternatively, we could define $\bar{\alpha}_{1}\left(k_{T}\right)$ to be the solution of the equation

$$
S_{1}\left(\bar{\alpha}_{1}, k_{T}, p_{T}\right) \equiv\left\langle S_{1}\left(\alpha, \vec{k}_{T}, \vec{p}_{T}\right) g\left(\alpha, k_{T}\right)\right\rangle_{\alpha}
$$

In practice there is only a percent or two difference between $\bar{\alpha}_{1}$ in the two definitions. Because $S_{1}\left(\alpha, \vec{k}_{T}, \vec{p}_{T}\right)$ is peaked about $k_{T}=p_{T}$, the same mean value of $\bar{\alpha}_{1}\left(k_{T}\right)$ characterizes, to a good approximation, the transverse momentum distribution both on the nucleon and the nucleus. Accordingly $\bar{\alpha}_{1}\left(k_{T}\right)$ is found to be essentially independent of A. We also find that for a given $x_{1}$ and $M, \bar{\alpha}_{1}\left(k_{T}\right)$ depends smoothly on $k_{T}$.

We show $\bar{\alpha}_{1}\left(k_{T}\right)$ for ${ }^{184} W$ in Fig. 4 for $x_{1}=0.46$, corresponding to E866. The quantity $\bar{\alpha}_{1}\left(k_{T}\right)$ shown there can be fitted by the function

$$
\bar{\alpha}_{1}\left(k_{T}\right)=\bar{\alpha}_{1}(0)+\left(a k_{T}+b k_{T}^{2}\right) /\left(1+c k_{T}^{2}\right) .
$$

The parameters in Eq. (39) are $\bar{\alpha}_{1}(0)=0.977, a=-0.0281, b=-0.0178$, and $c=$ 0.134. This function is relatively insensitive to $x_{2}$ over the range encompassed by the data. However, at larger $k_{T}, g\left(\alpha, k_{T}\right)$ spreads out sufficiently in $\alpha$ for $\bar{\alpha}_{1}\left(k_{T}\right)$ to acquire some sensitivity to the interval over which $\alpha$ is averaged in Eq. (37). Consequently, for larger $x_{1}$, $\bar{\alpha}_{1}\left(k_{T}\right)$ levels off at a smaller value. 


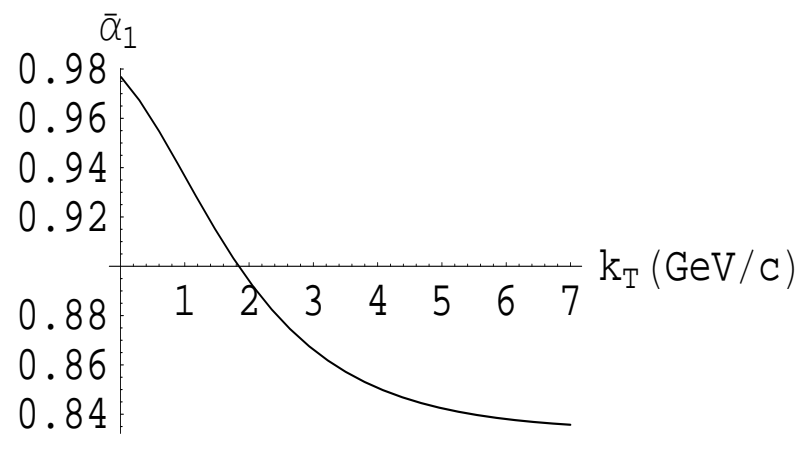

Figure 4: The quantity $\bar{\alpha}_{1}\left(k_{T}\right)$ of Eq. (38) for $800 \mathrm{GeV}$ protons incident on ${ }^{184} W$.

Because of the weak dependence of $\sigma_{0}$ on $s_{q},\left\langle S_{0}(\alpha)\right\rangle_{\alpha}$ in nearly independent of $p_{T}$. With $\left\langle S_{0}(\alpha)\right\rangle_{\alpha}$ and $\bar{\alpha}_{1}\left(k_{T}\right)$ so determined, our final result for the momentum distribution is given by

$$
\sigma_{D Y}^{p A}\left(p_{T}\right)=\left\langle\sigma_{D Y}^{q A}\left(\alpha, p_{T}\right)\right\rangle_{\alpha}=\left\langle S_{0}(\alpha)\right\rangle_{\alpha} \sigma_{D Y}^{p p}\left(p_{T}\right)+\int \frac{d^{2} k_{T}}{(2 \pi)^{2}} S_{1}\left(\bar{\alpha}_{1}\left(k_{T}\right), \vec{k}_{T}, \vec{p}_{T}\right) \sigma_{D Y}^{p p}\left(k_{T}\right)
$$

In the next section we evaluate Eq. (40) and compare it to the experimental results.

\subsection{Comparison to FermiLab E866 DY momentum distributions}

The theoretical calculations of this section correspond to the transverse momentum distribution $\sigma_{D Y}^{p A}\left(p_{T}\right)$ given in Eq. (27). The distributions are determined once the color dipole cross section, the nuclear density, and the gluon shadowing corrections are specified.

The gluon shadowing correction $R_{G}(x, A)$ is given in Fig. 4 of Ref. [15] as a function of $x$ for several values of $A$. In this paper we determine $R_{G}(x, A)$ for the nuclei of interest by extrapolating from the values given there. The value of $x$ at which we need to evaluate $R_{G}$ is determined by the square of the transverse momentum $k^{2}$ that a quark acquires scattering off the gluon cloud of a nucleon at quark-nucleon center of mass energy squared $s_{q}$ and is given by $x=4 k^{2} / s_{q}$. The average value of $k^{2}$ is fixed by the position of the peak of the effective gluon density in the KST model of the color-dipole cross section at $s_{q}$. For $s_{q}=500 \mathrm{GeV}^{2}$, we find $k^{2}=.5 \mathrm{GeV}^{2}[15]$ and accordingly $x=10^{-3}$. Although the gluon shadowing correction grows with the size of the target, our calculations show that the momentum distribution is rather insensitive to $R_{G}(x, A)$ for the SCL kinematics considered in this paper. 
The results that will be shown were evaluated using Eq. (40), which was obtained from Eq. (27) by a series of approximations, accurate at the few percent level, and made to facilitate the interpretation of the results and to streamline the numerical calculation of them. This calculation entails integrating the product of $S_{1}\left(\bar{\alpha}_{1}, p_{T}, k_{T}\right)$, given in Eq. (36), and $\sigma_{D Y}^{p p}\left(k_{T}\right)$ over $k_{T}$ using the parametrizations of $\bar{\alpha}_{1}\left(k_{T}\right)$ and $\sigma_{D Y}^{p p}\left(k_{T}\right)$. We will see in the next section that the transverse momentum dependence of $\bar{\alpha}_{1}\left(k_{T}\right)$ and $\sigma_{D Y}^{p p}\left(k_{T}\right)$ are key ingredients for understanding $\Delta\left\langle p_{T}^{2}\right\rangle$.

We begin our study with the examination of the E866 FermiLab fixed target experiment with an $800 \mathrm{GeV}$ proton beam. The relevant data are nuclear distribution ratios of Eq. (1) found in Refs. $[4,5,36]$. The cross sections appearing in these ratios are doubly differential distributions binned in $M^{2}$ (or $\left.x_{1}\right)$ and $x_{2}$,

$$
\sigma_{D Y}^{p A}\left(p_{T}\right) \equiv \frac{d^{4} \sigma_{D Y}^{p A}}{d M^{2} d x_{2} d^{2} k_{T}} .
$$

The individual cross sections that appear in these ratios were not determined as accurately as the ratios and are therefore not available. The data is binned in unique $x_{2}$ intervals within a tight range in $x_{1}$, i.e. $\left\langle x_{1}\right\rangle \approx 0.46[36]$.

Of these sets, those which have $0.1>x_{2}>0.05$ correspond to the short-coherence length limit, $\left\langle\ell_{c}\right\rangle<2 \mathrm{fm}$, where the momentum broadening is determined by initial state interactions, and it is this data set to which we compare our theory. For the E866 data corresponding to smaller $x_{2}$, the coherence length begins to exceed the internucleon spacing at the center of the nucleus. The coherence length remains at the same time smaller than the nuclear diameter for these data sets, so these data correspond to a situation where the SCL and LCL mechanisms are both relevant, requiring Green's function techniques or interpolation, as discussed earlier.

We would like to avoid data for which the LCL mechanism begins to play a role. The reason is that the mean-square transverse momentum corresponding to the LCL mechanism is poorly defined [29]. Although our recent analysis in Ref. [8] found no evidence for the mean-square momentum to depend on coherence length, the large statistical errors do not rule out as much as a factor of two variation among the various data sets. Thus, we restrict our study to those momentum distribution ratios that correspond to short coherence length with $0.1>x_{2}>0.05$ and for which the measured broadening of dileptons is related to soft initial state interactions and broadening of the projectile quark.

For $\sigma_{D Y}^{p p}\left(k_{T}\right)$ we have chosen a parametrization similar to that in Ref. [32],

$$
\sigma_{D Y}^{p p}\left(p_{T}\right)=N \frac{\left(1+\frac{p_{T}^{2}}{\lambda_{1}^{2}} e^{-p_{T}^{2} / \lambda_{2}^{2}}\right)}{\left(1+p_{T}^{2} / \lambda_{3}^{2}\right)^{n}},
$$

where the factor in the numerator is introduced to describe a possible forward minimum in the cross section indicated by data [22]. It was found that the shape of the $p_{T}$ dependence does not vary with dilepton mass and $x_{2}$. A global fit to Drell-Yan data from $p p$ collisions measure by the E866 experiment for different $M$ and $x_{2}$ bins [22], with common parameters $n, \lambda_{i}$ and different normalization factors $N$, led to quite a good description [40], $\chi^{2} /$ d.o.f. $=$ 


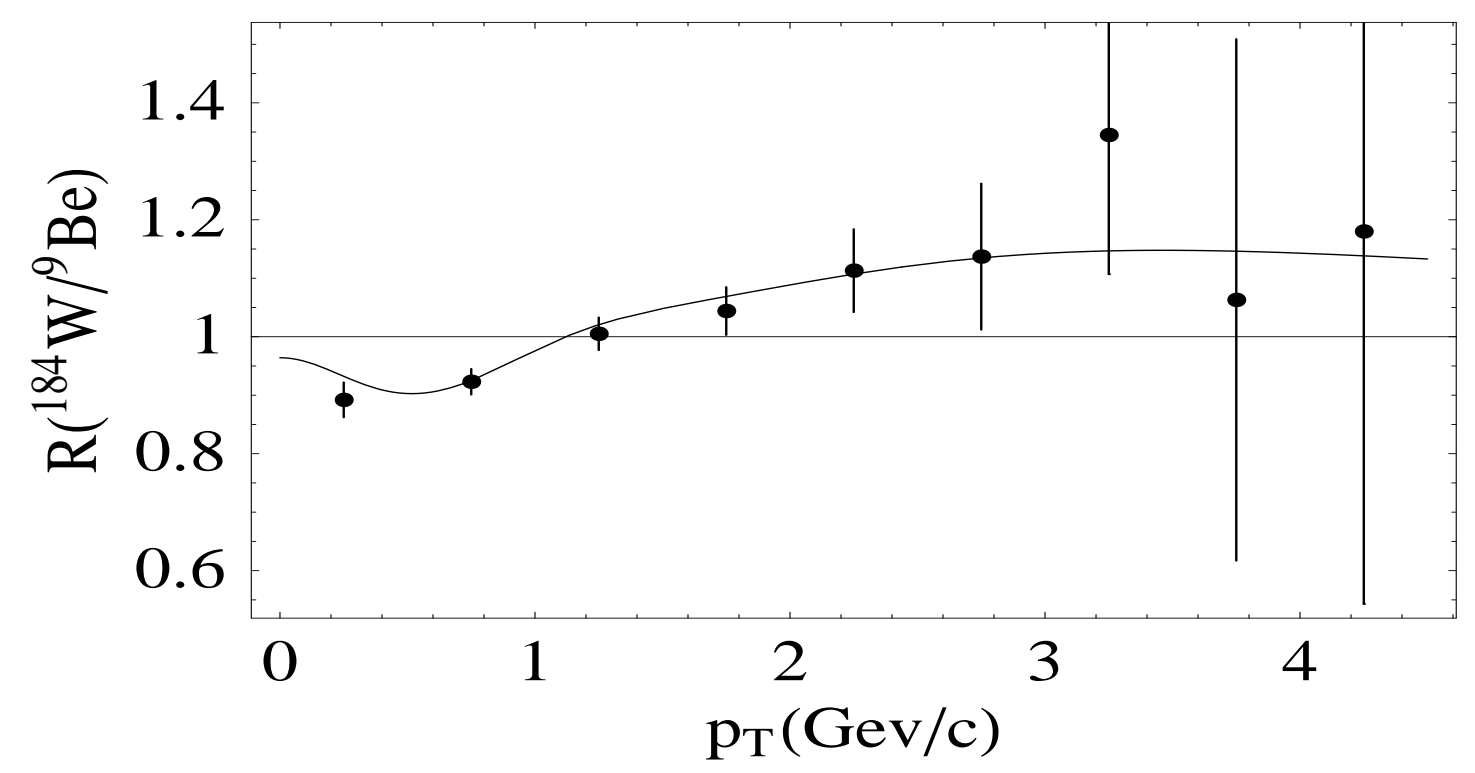

Figure 5: Comparison of the theoretical prediction for $R^{W / B e}\left(p_{T}\right)$ vs. $p_{T}$ (in $G e V / c$ ) with experiment for $x_{2}=0.05$. Data are from the FermiLab E772/E866 collaboration [4, 5,36].

1.5. The values of the parameters are $\lambda_{1}=0.74 \pm 0.12 ; \lambda_{2}=0.59 \pm 0.06 ; \lambda_{3}=3.03 \pm 0.25$; $n=6.24 \pm 0.74$.

Notice that Eq. (25) is not the same as $\sigma_{D Y}^{p p}\left(p_{T}\right)$ calculated as in Eq. (10). By using the phenomenological proton-proton transverse momentum distribution we compensate for the fact that a portion of the transverse momentum distribution arises from the density matrix of the initial quark in the projectile that is not yet accounted for in Eq. (10). This explains why the measured DY momentum distribution falls off more slowly with transverse momentum than Eqs. (9) and (10) evaluated as explained in the text.

Our prediction for $R^{W / B e}\left(p_{T}\right)$ is shown in Fig. 5. For this calculation, the gluon shadowing correction was determined to be $R_{G}=0.9$ for ${ }^{184} \mathrm{~W}$ and $R_{G}=1.0$ for ${ }^{9} \mathrm{Be}$. One sees that the theory is in quite good agreement with experiment. Notice that the details

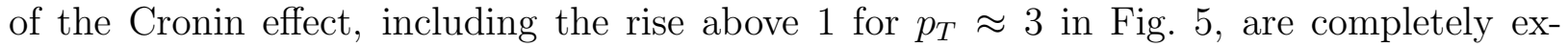
plained with no adjustable parameters. For $W$, the value of $\left\langle T_{A}>=1.35\right.$, and for $B e$ it is $<T_{A}>=0.288$. We use densities from Ref. [41].

The importance of the momentum dependence of $\bar{\alpha}_{1}\left(k_{T}\right)$ is illustrated in Fig. [6, where we compare the calculation of $R^{W / p}$ with $\bar{\alpha}_{1} \rightarrow \bar{\alpha}_{1}\left(k_{T}=0\right)$ with the same quantity, but now with $\bar{\alpha}_{1}\left(k_{T}\right)$. We see that retaining the dependence on $k_{T}$ makes $R^{W / p}$ drop somewhat faster at larger values of $p_{T}$.

Our prediction for $R^{\mathrm{Fe} / \mathrm{Be}}\left(p_{T}\right)$ is shown in Fig. 7. For the calculation, we found the 


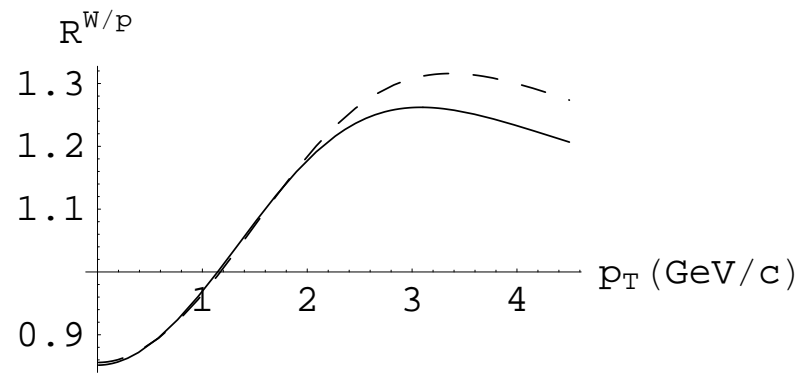

Figure 6: $R^{W / p}\left(p_{T}\right)$ for ${ }^{184} W$ with $\bar{\alpha}_{1}=\bar{\alpha}_{1}\left(p_{T}\right)$ (solid curve), and with $\bar{\alpha}_{1}=\bar{\alpha}_{1}\left(p_{T}=0\right)=0.9775$ (dashed curve).

gluon shadowing correction to be $R_{G}=0.95$ for $F e$. We find comparable agreement for both $R^{W / B e}$ and $R^{F e / B e}$.

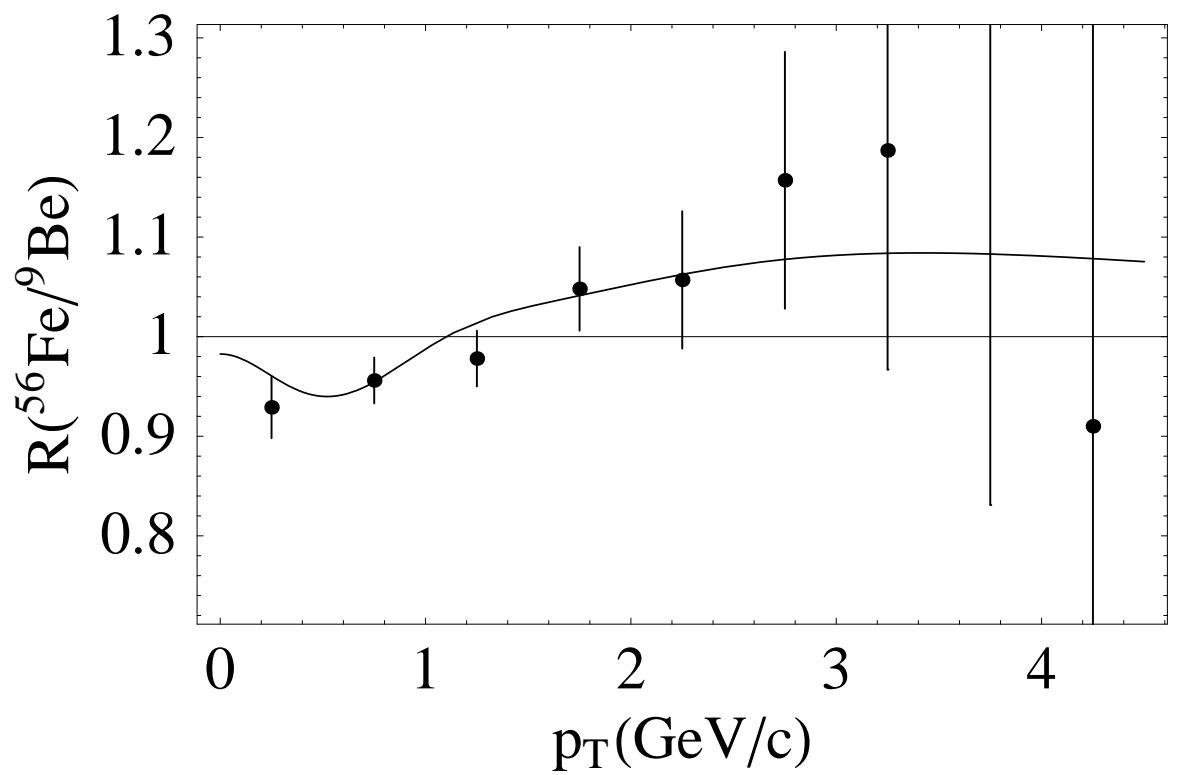

Figure 7: Comparison of the theoretical prediction for $R^{F e / B e}\left(p_{T}\right)$ vs. $p_{T}$ (in $\mathrm{GeV} / \mathrm{c}$ ) with experiment for $x_{2}=0.05$. Data are from the FermiLab E772/E866 collaboration [4,5,36]. 


\subsection{Transverse momentum broadening}

The transverse momentum broadening for a dilepton produced on a nucleus is defined in Eq. (2) where the mean momentum is defined as,

$$
\left\langle p_{T}^{2}\right\rangle=\frac{\int d p_{T}^{2} p_{T}^{2} \sigma_{D Y}^{p A}\left(p_{T}\right)}{\int d p_{T}^{2} \sigma_{D Y}^{p A}\left(p_{T}\right)} .
$$

The dependence of the broadening $\Delta\left\langle p_{T}^{2}\right\rangle$ on $A$ for the production of heavy lepton pairs has been studied experimentally using the E772 data in Ref. [7], which was revised recently in [8] with the inclusion of the E866 data. It was found that for large A

$$
\Delta\left\langle p_{T}^{2}\right\rangle_{\text {exp }}^{A}=D(A / 2)^{1 / 3} \mathrm{GeV}^{2},
$$

where the factor $D$ was found to be for the two sets of data,

$$
\begin{gathered}
D(E 772)=(0.029 \pm 0.008+0.009)(\mathrm{GeV} / c)^{2} \\
D(E 866)=(0.059 \pm 0.009+0.01)(\mathrm{GeV} / c)^{2}
\end{gathered}
$$

The last correction here is the systematic error, with a sign known to be positive. This correction, if added, must be applied to both E772 and E866 simultaneously. It comes from the theoretical uncertainty related to the lack of data at large $p_{T}$.

We next calculate $\Delta\left\langle p_{T}^{2}\right\rangle$ in the theory just described. Because the evaluation of $\left\langle p_{T}^{2}\right\rangle$ in Eq. (43) entails an integration over all transverse momenta, we are lead to delta functions that require evaluations of various quantities at $\vec{r}_{T}=0$. For this reason, we begin by transforming to coordinate space. From Eq. (29) we easily find that

$$
\sigma_{D Y}^{p A}\left(p_{T}\right)=\left\langle\sigma_{D Y}^{q p}\left(\alpha, r_{T}\right)\right\rangle_{\alpha}=\frac{1}{A} \int d^{2} r_{T} e^{i \vec{p}_{T} \cdot \vec{r}_{T}} e^{-\frac{\alpha^{2} r_{T}^{2}}{6\left\langle r_{c m}^{2}\right\rangle}} U\left(\alpha r_{T}\right)\left\langle\sigma_{D Y}^{q p}\left(\alpha, r_{T}\right)\right\rangle_{\alpha}
$$

where $U\left(r_{T}\right)$ is given in Eq. (30) and

$$
\left\langle\sigma_{D Y}^{q p}\left(\alpha, r_{T}\right)\right\rangle_{\alpha} \equiv \sigma_{D Y}^{p p}\left(r_{T}\right)=\int \frac{d^{2} p_{T}}{(2 \pi)^{2}} e^{-i \vec{p}_{T} \cdot \vec{r}_{T}}\left\langle\sigma_{D Y}^{q p}\left(\alpha, p_{T}\right)\right\rangle_{\alpha}
$$

Multiplying Eq. (46) by $p_{T}^{2}$ and integrating over all $\vec{p}_{T}$, we find, using the fact that $\sigma_{\bar{q} q}\left(r_{T}\right)$ is proportional to $r_{T}^{2}$ at small $r_{T}$ (see Eq. (8) ),

$$
\left\langle p_{T}^{2}\right\rangle^{A}=\left\langle p_{T}^{2}\right\rangle^{N}+\frac{4 C N\left\langle\alpha^{2}\right\rangle}{2 A} \int d^{2} b \int d z \int d^{2} R \rho(R-b, z) e^{-\frac{2 R^{2}}{3\left\langle r_{c m}^{2}\right\rangle}} T_{A}(b, z),
$$

where

$$
\left\langle p_{T}^{2}\right\rangle^{N}=-\left.\frac{\nabla^{2} \sigma_{D Y}^{p p}\left(r_{T}\right)}{\sigma_{D Y}^{p p}\left(r_{T}\right)}\right|_{r_{T}=0}+\frac{2}{3} \frac{\left\langle\alpha^{2}\right\rangle}{\left\langle r_{c m}^{2}\right\rangle}
$$

is the mean transverse momentum of DY reaction on a proton target, and where

$$
\left\langle\alpha^{2}\right\rangle=\frac{1}{\left\langle\sigma_{D Y}^{q p}\left(\alpha, r_{T}=0\right)\right\rangle_{\alpha}}\left\langle\alpha^{2} \sigma_{D Y}^{q p}\left(\alpha, r_{T}=0\right)\right\rangle_{\alpha}
$$


The quantity $\left\langle\alpha^{2}\right\rangle$ in Eq. (50) is the fraction of the light cone momentum carried by the parton of the projectile that radiates the $\gamma *$, averaged over the transverse momentum for fixed $x_{1}$, as can be seen by transforming back to momentum space. Using the Fourier transform in Eq. (47) and the definition of $\sigma_{D Y}^{q p}\left(\alpha, k_{T}\right)$ in Eq. (26), with the quantity $\bar{\alpha}_{1}\left(p_{T}\right)$ shown in Fig. 6 as defined in Eq. (37), Eq. (50) may be equivalently written as

$$
\left\langle\alpha^{2}\right\rangle=\frac{\int d^{2} p_{T} \sigma_{D Y}^{p p}\left(p_{T}\right) \bar{\alpha}_{1}\left(p_{T}\right)^{2}}{\int d^{2} p_{T} \sigma_{D Y}^{p p}\left(p_{T}\right)} .
$$

We find that $\left\langle\alpha^{2}\right\rangle \approx 0.86$. This value is slightly smaller than the previous estimates in Ref. [29] $\left(\left\langle\alpha^{2}\right\rangle \approx 0.9\right)$ because the integrand in Eq. (51) peaks at slightly higher $p_{T}$ due to the fact that the phenomenological momentum distribution of the proton $\sigma_{D Y}^{p p}\left(p_{T}\right)$ is softer than the theoretical distribution used in Ref. [29] (see discussion below Eq. (25)).

The first term in Eq. (49) corresponds to the contribution to the mean transverse momentum arising from the hard interaction, and the second term is the contribution arising from the primordial transverse momentum of a quark in the projectile hadron. The contribution to $\left\langle p_{T}^{2}\right\rangle^{N}$ arising from the hard interaction is formally infinite. As long as there is no shadowing, as in the SCL, this gets subtracted to obtain $\Delta\left\langle p_{T}^{2}\right\rangle$,

$$
\Delta\left\langle p_{T}^{2}\right\rangle \equiv\left\langle p_{T}^{2}\right\rangle^{A}-\left\langle p_{T}^{2}\right\rangle^{N}=\frac{4 C N\left\langle\alpha^{2}\right\rangle}{2 A} \int d^{2} b \int d z \int d^{2} R \rho(\vec{R}-\vec{b}, z) e^{-\frac{2 R^{2}}{3\left\langle r_{c m}^{2}\right\rangle}} T_{A}(b, z) .
$$

This cancellation is not complete for the LCL case [29], but we have avoided the associated complications by restricting our attention to $x_{2}>0.05$, where the SCL dominates. Using Eq. (52) and dropping the center-of-mass density contribution (the second term in Eq. (A.8) of the appendix), which is only about a $3 \%$ correction here, we find

$$
\Delta\left\langle p_{T}^{2}\right\rangle=\left\langle\alpha^{2}\right\rangle C\left\langle T_{A}\right\rangle
$$

where we have used the average of $T_{A}$ as given in Eq. (32). We recall [15] in passing that Eq. (53) for broadening of a dilepton pair produced in DY is closely related to the broadening experienced by a quark propagating through nuclear matter, differing importantly by the following considerations. First, on average a quark in the SCL limit passes through only half the nuclear thickness before it produces the dilepton pair (thus Eq. (53) is missing the factor of 2 that appears in Eq. (20) of Ref. [15]). Secondly, the quark gives up only a fraction $\alpha$ of its transverse momentum to the dilepton pair, which accounts for the extra factor of $\left\langle\alpha^{2}\right\rangle$ in Eq. (53).

We may now compare with the experimental result in Eq. (44). Using values of $C=4.475$ corresponding to the KST model used for our calculations of the momentum distributions, and taking the uniform density model for the nuclear density,

$$
\left\langle T_{A}\right\rangle=\frac{3}{2} \rho_{0} R_{A}
$$

where $\rho_{0} \approx 0.16 \mathrm{fm}^{-3}$ is the central density of heavy nuclei and $R_{A} \approx 1.1 A^{\frac{1}{3}}$, we find from theory that

$$
\Delta\left\langle p_{T}^{2}\right\rangle_{T h}^{A} \approx 0.049(A / 2)^{\frac{1}{3}} \mathrm{GeV}^{2}
$$


or $D=.049$. This agrees with the central value of $D(E 866)$ given in Eq. (45) to within the statistical plus systematic errors and is only slightly outside the errors on $D(E 772)$. It was concluded in Ref. [8] that large systematic errors must be assigned to the value of $D$ in the original $E(772)$ analysis, and our results in Eq. (55) are certainly consistent with this observation.

\subsection{Predictions for transverse momentum observables in the SCL at RHIC}

Since momentum broadening in DY uniquely characterizes the momentum broadening of the quark in the initial state, a measurement of DY at RHIC would complement information obtained from momentum distributions obtained from production of pions, which are additionally sensitive to final state interactions. As seen in Fig. 3, the SCL prevails at RHIC for DY in proton-nucleus collisions when $x_{2}^{R H I C}>0.05$, for a range of transverse momentum up to several $\mathrm{GeV} / \mathrm{c}$. This regime at $x_{F}=x_{1}-x_{2}=0$ is not easily accessed experimentally with current detectors at RHIC, but for the reason given before, it would be interesting to measure. The light-cone target-rest-frame formulation provides a theoretical framework for calculating the transverse momentum observables for RHIC, and we next use this theory for making predictions.

In $d A$ collisions at the RHIC collider beams of deuterons are incident on nuclei at an energy of $100 \mathrm{GeV} / \mathrm{N}$, corresponding to collisions between the projectile proton with a nucleon in the nucleus at $\sqrt{s}=200 \mathrm{GeV}$. In the target rest frame, this corresponds to protons incident on the nucleus with laboratory energy of about $E_{p}^{R H I C}=21 \mathrm{TeV}$. However, for $x_{1}^{R H I C}=.05$, corresponding to the smallest value of Bjorken $x_{1}$ at which the SCL limit prevails, the quarks of the beam proton that radiate the dilepton pair have an average energy of only about $\left\langle E_{q}^{R H I C}\right\rangle=x_{1}^{R H I C} E_{q}^{R H I C}=1.1 \mathrm{TeV}$, which is not so different from the average quark energy in the E866 FermiLab experiments. As indicated earlier, the FermiLab E866 experiment involves larger $x_{1} \approx 0.46$, which is mainly responsible for the small differences we predict.

For RHIC, we will do our calculation for the lowest value of $x_{1}$ for which the SCL prevails, $x_{1}=x_{2}=0.05$. For this value of $x_{1}$, we find that the dependence of $\bar{\alpha}_{1}\left(k_{T}\right)$ is given by Eq. (39) with $\bar{\alpha}(0)=0.9513 a=0.0168, b=-0.137$, and $c=0.197$, corresponding to a somewhat stronger fall-off of $\bar{\alpha}_{1}\left(k_{T}\right)$ with $k_{T}$ than we found for E866.

For gluon shadowing, the larger RHIC energies do not lead to much different $R_{G}(x, A)$ from the values found for heavy nuclei at FermiLab. For determining the effective value of $x=4 k^{2} / s_{q}$ [15] for $R_{G}(x, A)$, we take $s_{q}=2 m E_{q}^{R H I C}=2000 \mathrm{GeV}^{2}$, corresponding to $k^{2}=.6 \mathrm{GeV}^{2}$. This gives $x=1.110^{-3}$ and $R_{G}=0.91$ from Fig. 4 of Ref. [15].

The transverse momentum distribution for protons on gold, according to our theory, is shown in Fig 8 . We see a clear Cronin peak at $p_{T}=2 \mathrm{GeV}$ of about the same size seen in DY ratios at FermiLab and in pion production at RHIC. The ratio to protons was calculated assuming that the transverse momentum dependence of DY production on protons is the same as measured in E866 at FermiLab and given in Eq. (42). One should of course use one more appropriate for the RHIC kinematics, but this is presently unknown. 
Using the distribution measured in E866 is reasonable since the kinematics are similar and presumably differences will tend to cancel in taking the ratio, but still there may be quantitative differences when the result is calculated with a known momentum distribution on the proton.

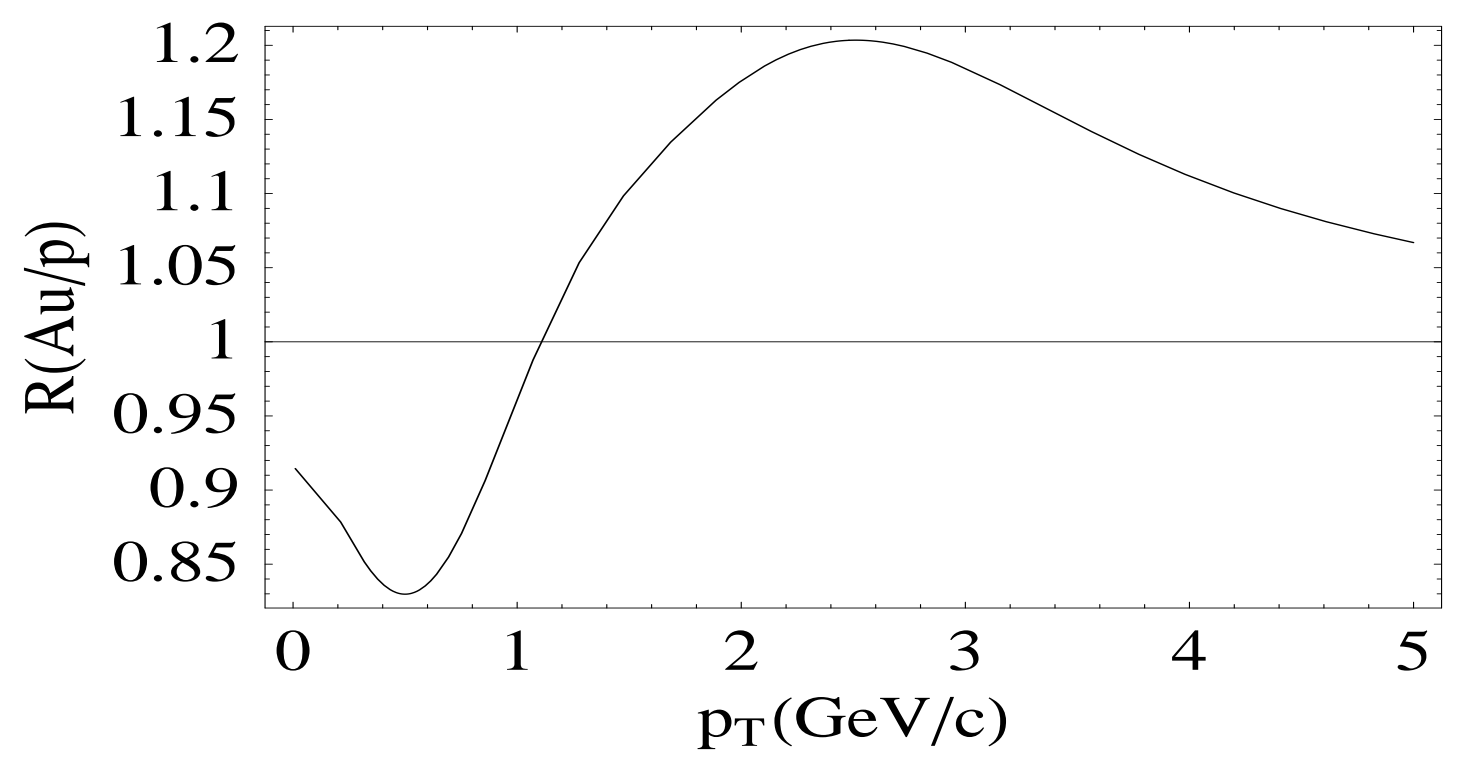

Figure 8: Theoretical prediction of $R^{A u / p}\left(p_{T}\right)$ vs. $p_{T}$ (in $G e V / c$ ) for $D Y$ at $\mathrm{RHIC}, \sqrt{s}=200 \mathrm{GeV}$ for $x_{F}=0\left(x_{2}=0.05\right)$.

The value of $\Delta\left\langle p_{T}^{2}\right\rangle$ corresponding to the distribution given in Fig. 8 is given by Eq. (53) with $\left\langle\alpha^{2}\right\rangle=0.67, C\left(s_{q}\right)=6.04$, and $\left\langle T_{A}\right\rangle=1.5$. The value of $\left\langle\alpha^{2}\right\rangle$ is somewhat smaller than it was for E866 since $\bar{\alpha}_{1}\left(k_{T}\right)$ falls off more quickly in $k_{T}$ because of the smaller $x_{1}$. This decrease (accidentally) almost completely compensates the increase in the transverse momentum acquired by the quark before it produces the dilepton pair, which is determined by $C\left(s_{q}\right)$ from the KST color-dipole cross section at $r_{T}=0$ and has a weak logarithmic increase with energy. We find

$$
\Delta\left\langle p_{T}^{2}\right\rangle_{T h}^{A} \approx 0.042(A / 2)^{\frac{1}{3}} \mathrm{GeV}^{2}
$$

corresponding to $D=0.042$, very close to our theoretical value of $D$ corresponding to E866 and given in Eq. (55).

\section{Summary and conclusions}

The color dipole approach formulated in the target rest frame provides a natural framework for examining nuclear modifications to parton propagation in collisions of hadronic 
projectiles on nuclei. In this paper, we are interested in transverse momentum broadening of fast quarks propagating in nuclei, which we have examined in a particular kinematic regime using the color dipole approach. The color-dipole approach has predictive power since it relates transverse momentum observables in nuclear collisions to the color-dipole cross section determined from deep-inelastic scattering on a nucleon target. Once the colordipole cross section is determined, predictions for these observables can be made with no parameters to be adjusted [15]. Transverse momentum broadening in Drell-Yan production has been recently measured with good accuracy in $p+A$ collisions in the E866 experiment at FermiLab $[4,5,36]$ with $E_{p}=800 \mathrm{GeV}$. In this paper, we have applied our theory to the FermiLab data.

In the short coherence length limit, $\ell_{c} \ll R_{A}$, energy loss and momentum broadening are mediated by multiple interactions of the incident quark with target nucleons before the emission of the $\gamma^{*}$. The SCL limit is appropriate for the FermiLab data and for application of the theory given in Ref. [15]. The transverse momentum distributions we calculate from this theory, presented in Sect. 3.3, are in good agreement with the measured ones. The value of $\Delta\left\langle p_{T}^{2}\right\rangle$ determined from the same theory gives the result presented in Eq. (55), which is comparable to the empirical determination of the same quantity $[7,8]$.

We have thus confirmed within the model studied that transverse momentum broadening in the SCL arises from soft initial state interactions experienced by the parton initiating the hard reaction, as described in the color-dipole approach. In addition, our results support the validity of the method of determining the mean-square transverse momentum distribution as given in Ref. [8].

Predictions for transverse momentum observables at RHIC in the short coherence limit have been presented, complementing our earlier study [29] of broadening in the long coherent length regime.

\section{Acknowledgements}

We would like to acknowledge our collaborators I.K. Potashnikova, A.V. Tarasov, J. Raufeisen, M. Leitch, J. Moss, and P. McGaughey. This work was supported in part by U.S. Department of Energy, the Research Ring "Center of Subatomic Studies" (Chile), Fondecyt (Chile) grant 1050519, and by DFG (Germany) grant PI182/3-1. 


\section{Appendix A: Average over nuclear density}

In this appendix, we are interested in computing the average over the nuclear density in the expression for the momentum distribution of a DY pair produced on a nucleus, $\sigma_{D Y}^{p A}\left(p_{T}\right)$,

$$
\sigma_{D Y}^{p A}\left(p_{T}\right)=\int d^{2} k_{T}\left\langle\left\langle W^{q}\left(k_{T}, s_{q}\right)\right\rangle_{N} \sigma_{D Y}^{q p}\left(\alpha, \vec{p}_{T}-\alpha \vec{k}_{T}\right)\right\rangle_{\alpha}
$$

For the purpose of evaluating the nuclear average, we note that the $\alpha$ dependence in $\sigma_{D Y}^{q p}\left(\alpha, p_{T}\right)$, as given by Eq. (10), is sharply peaked around $\alpha=1$. This means that it is justified to replace the more slowly-dependent functions of $\alpha$ by averages. One source of $\alpha$ dependence is the quark energy $s_{q}$ that appears in $W^{q}\left(k_{T}, s_{q}\right)$ through the KST colordipole cross section. In the target rest frame, $s_{q}$ is related to the quark energy $E_{q}$ by $s_{q}=2 m_{p} E_{q}$, with $E_{q}=E_{p} x_{1} / \alpha$. As the color-dipole cross section depends only weakly on $s_{q}$, the average is insensitive to the specific value, which on average is about $\bar{E}_{q} \approx E_{p} x_{1}$. The remaining $\alpha$ dependence is the explicit factor appearing in Eq. (A.1).

Then, the momentum distribution $\sigma_{D Y}^{p A}\left(p_{T}\right)$ may thus be expressed, to an excellent approximation, as

$$
\begin{aligned}
\sigma_{D Y}^{p A}\left(p_{T}\right) & \approx \int d^{2} k_{T}\left\langle W^{q}\left(k_{T}, \bar{s}_{q}\right)\right\rangle_{N}\left\langle\sigma_{D Y}^{q p}\left(\alpha, \vec{p}_{T}-\alpha \vec{k}_{T}\right)\right\rangle_{\alpha} \\
& \approx \int d^{2} k_{T} W^{q A}\left(k_{T}, \bar{s}_{q}\right) \sigma_{D Y}^{p p}\left(\vec{p}_{T}-\bar{\alpha} \vec{k}_{T}\right)
\end{aligned}
$$

where $\bar{\alpha}_{1}$ may depend on $k_{T}$, i.e. $\bar{\alpha}_{1}=\bar{\alpha}_{1}\left(k_{T}\right)$ and where $\sigma_{D Y}^{p p}\left(k_{T}\right)$ is defined as in Eq. (25)). Using Eq. (17) in Eq. (A.3) and changing variables, we may obtain expressions for $\sigma_{D Y}^{p A}\left(p_{T}\right)$ and $U\left(r_{T}\right)$ identical to those in Eqs. (29),

$$
\begin{gathered}
\sigma_{D Y}^{p A}\left(p_{T}\right)=\frac{1}{A} \int \frac{d^{2} k_{T}}{(2 \pi)^{2}} \int d^{2} r_{T} e^{i \vec{k}_{T} \cdot \vec{r}_{T}} e^{-\frac{r_{T}^{2}}{6\left\langle r_{c m}^{2}\right\rangle}} U\left(r_{T}\right) \sigma_{D Y}^{p p}\left(\vec{p}_{T}-\bar{\alpha} \vec{k}_{T}\right) \\
U\left(r_{T}\right)=N \int d^{2} b \int d z \int d^{2} R \rho_{A}(\vec{R}-\vec{b}, z) e^{-\frac{2 R^{2}}{3\left\langle r_{c m}^{2}\right\rangle}} e^{-\frac{1}{2} \sigma_{\bar{q} q}\left(r_{T}, \bar{s}_{q}\right) T_{A}(b, z)} .
\end{gathered}
$$

except that $\bar{\alpha}_{1}$ and $\bar{s}_{q}$ replace $\alpha$ and $s_{q}$.

In this appendix, we calculate $\sigma_{D Y}^{p A}\left(p_{T}\right)$ in specific models, and show that we are able to get quantitative agreement with the exact expression, Eq. (A.5), if we make the average thickness function prescription of Eq. (31) for the model.

One model we consider is the uniform density model,

$$
\rho_{A}(b, z)=\rho_{0} \theta\left(R_{0}-r\right)
$$

where $R_{0}$ and $\rho_{0}$ are fixed to preserve $A$ and $\left\langle T_{A}\right\rangle$ of a realistic density for the nucleus in question. We find in practice that when $R_{0}$ and $\rho_{0}$ are fixed in this fashion, the uniform density model can be applied to calculate $\sigma_{D Y}^{p A}$ for nuclei as light as ${ }^{9} B e$. For light nuclei, we use a gaussian density,

$$
\rho_{A}(b, z)=\rho_{0} e^{-\left(b^{2}+z^{2}\right) / R_{0}^{2}} .
$$


where $R_{0}$ and $\rho_{0}$ are again fixed to preserve $A$ and $\left\langle T_{A}\right\rangle$ of a realistic density.

To calculate $U\left(r_{T}\right)$, we begin by writing

$$
\rho_{A}(\vec{R}-\vec{u}, z)=\rho_{A}(u, z)+\delta \rho_{A}(\vec{R}, \vec{u}, z)
$$

and noting that $U\left(r_{T}\right)$ may be written

$$
U\left(r_{T}\right)=U_{0}\left(r_{T}\right)+\delta U\left(r_{T}\right)
$$

where

$$
U_{0}\left(r_{T}\right)=\int d^{2} b \int_{0}^{T_{A}(b)} d T_{A} e^{-\frac{1}{2} \sigma_{\bar{q} q}\left(r_{T}, \bar{s}_{q}\right) T_{A}}
$$

and

$$
\delta U\left(r_{T}\right)=N \int d^{2} u \int d z \int d^{2} R \delta \rho_{A}(\vec{R}, \vec{u}, z) e^{-\frac{2 R^{2}}{3\left\langle r_{c m}^{2}\right\rangle}} e^{-\frac{1}{2} \sigma_{\bar{q} q}\left(r_{T}, \bar{s}_{q}\right) T_{A}(u, z)} .
$$

We refer to Eq. A.11 as the center-of-mass correction.

In general, $U\left(r_{T}\right)$ is characterized by a set of numbers $U_{L}$,

$$
U\left(r_{T}\right)=\sum_{L=0} U_{L} e^{-L r_{T}^{2} / R_{0}^{2}} / L !
$$

obtained by expanding $e^{-r_{T}^{2} / R_{0}^{2}}$ in $\sigma_{\bar{q} q}\left(r_{T}, \bar{s}_{q}\right)$ (see Eq. (77)). With this expansion, we may do the integrals over $r_{T}$ in Eq. (A.5), leading to

$$
\sigma_{D Y}^{p A}\left(p_{T}\right)=\frac{1}{A} \sum_{L=0} \frac{\pi}{\frac{L}{R_{0}^{2}}+\frac{1}{6\left\langle r_{c m}^{2}\right\rangle}} \frac{U_{L}}{L !} \int \frac{d^{2} k_{T}}{\bar{\alpha}_{1}^{2}(2 \pi)^{2}} e^{-\frac{k_{T}^{2}}{4 \bar{\alpha}_{1}^{2}} \frac{L}{\frac{L}{R_{0}^{2}}+\frac{1}{6\left\langle r_{c m}^{2}\right\rangle}}} \sigma_{D Y}^{p p}\left(\vec{p}_{T}-\vec{k}_{T}\right) .
$$

In the uniform density model, $U_{0 L}$, corresponding to the first term in Eq. (A.9), may be worked out analytically to give

$$
U_{0 L}=\frac{2 \pi R_{0}^{2}}{c^{2} \sigma_{0}}\left[\Gamma(L+3, c)-\Gamma(L+3)+c^{2} \Gamma(L+1)-c^{2} \Gamma(L+1, c)\right],
$$

where

$$
c=\frac{2}{3} \sigma_{0}\left\langle T_{A}\right\rangle
$$

with $\left\langle T_{A}\right\rangle$ defined in Eq. (32). For the gaussian density, $U_{0 L}$ becomes

$$
U_{0 L}=A / c \int_{0}^{\infty} d x\left(\Gamma(L+1)-\Gamma\left(L+1, c e^{-x}\right)\right)
$$

where now

$$
c=\sigma_{0}\left\langle T_{A}\right\rangle \text {. }
$$

The contribution $\delta U_{L}$ of the center-of-mass correction for both the uniform density model and the gaussian model have been evaluated numerically. For the average thickness function prescription, $U_{L}$ may be read from Eq. (33),

$$
U_{L}=A e^{\frac{\sigma_{0}}{2}\left\langle T_{A}\right\rangle / 2}\left(\frac{\sigma_{0}}{2}\left\langle T_{A}\right\rangle / 2\right)^{L},
$$


with no center-of-mass correction.

We next illustrate the validity of the average thickness function prescription of Eq. (32) for a heavy nucleus $(A=184)$ in the uniform density model and a light nucleus $(A=9)$ in the gaussian density model. In these cases, we evaluate $\sigma_{D Y}^{p A}\left(p_{T}\right)$ as given in Eq. (A.13) for $\bar{s}_{q}=600 \mathrm{GeV}$ and $\bar{\alpha}=1$, but similar results are obtained for energies relevant at RHIC and for the full range of $\bar{\alpha}$ that enters into the calculation of the nuclear momentum distribution in Fig. 6,

For $A=184$, we take $\left\langle T_{A}\right\rangle=1.35$, which corresponds to a nuclear density proportional to the charge density for ${ }^{184} W$ [41]. The parameters of the uniform density (UD) model are then $R_{0}=6.99 \mathrm{fm}$ and $\rho_{0}=0.129 \mathrm{fm}^{-3}$. The result of calculating $R_{U D}^{W / p}$, including the contributions from $U_{0 L}$ of Eq. (A.14) and $\delta U_{L}$ calculated numerically, is shown in Fig. 9. In

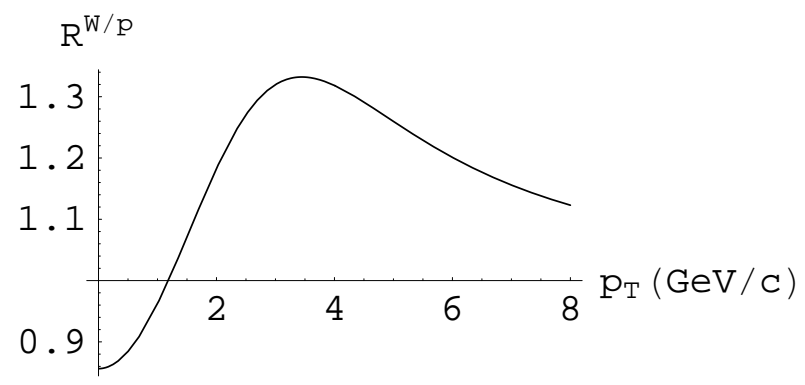

Figure 9: Nuclear ratio $R^{W / p}\left(p_{T}\right)$ in the uniform density model calculated using Eq. (A.13), including the center-of-mass correction.

Fig. 10, the result in Fig. 9 is compared to the corresponding quantity calculated using the average thickness function (ATF) prescription, $R_{A T F}^{W / p}$; specifically, the solid curve gives

$$
100 \frac{R_{U D}^{W / p}\left(p_{T}\right)-R_{A T F}^{W / p}\left(p_{T}\right)}{R_{U D}^{W / p}\left(p_{T}\right)} .
$$

Clearly, $R_{U D}^{W / p}$ and $R_{A T F}^{W / p}$ are equal to about one percent or better. The dashed curve in Fig. 10 shows the contribution of the center-of-mass correction $\delta R_{U D}^{W / p}$ to $R_{U D}^{W / p}$; specifically, the dashed curve gives

$$
100 \frac{\delta R_{U D}^{W / p}\left(p_{T}\right)}{R_{U D}^{W / p}\left(p_{T}\right)} .
$$

The center-of-mass correction is clearly quite small, less than a percent. 


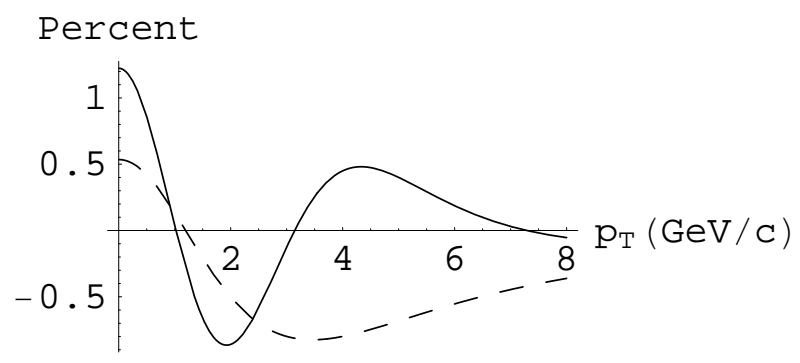

Figure 10: Comparison of $R_{U D}^{W / p}$ and $R_{A T F}^{W / p}$ (solid curve); relative contribution of center-of-mass correction to $R_{U D}^{W / p}$ in the uniform density model (dashed curve). See text for details.

For $A=9$, we take $\left\langle T_{A}\right\rangle=0.288$, which corresponds to a nuclear density proportional to the charge density for ${ }^{9} B e$ [41]. The parameters of the gaussian density (GD) model are then $R_{0}=2.23 \mathrm{fm}$ and $\rho_{0}=0.146 \mathrm{fm}^{-3}$. The result of calculating $R_{G D}^{W / p}$ including the contributions from $U_{0 L}$ of Eq. (A.16) and $\delta U_{L}$ calculated numerically is shown in Fig. 11. We note in passing that essentially the same results for ${ }^{9} \mathrm{Be}$ are found when we apply the uniform density model to this nucleus. The solid curve in Fig. 12 compares $R_{G D}^{B e / p}$ to the corresponding quantity in the average thickness function prescription, $R_{A T F}^{B e / p}$, specifically,

$$
100 \frac{R_{G D}^{B e / p}\left(p_{T}\right)-R_{A T F}^{B e / p}\left(p_{T}\right)}{R_{G D}^{B e / p}\left(p_{T}\right)} .
$$

As in the case of ${ }^{184} W, R_{U D}^{B e / p}$ and $R_{A T F}^{B e / p}$ are equal to about one percent or better. The contribution of the center-of-mass correction $\delta R_{G D}^{B e / p}$ to $R_{G D}^{B e / p}$, specifically,

$$
100 \frac{\delta R_{G D}^{B e / p}\left(p_{T}\right)}{R_{G D}^{B e / p}\left(p_{T}\right)}
$$

is shown as the dashed curve in Fig. 12. The center-of-mass correction for ${ }^{9} \mathrm{Be}$ is clearly quite small; as for ${ }^{184} W$, it contributes less than a percent to the nuclear ratio. Because the center-of-mass correction in Eq. (A.11) is very small even for light nuclei, it will remain small for all realistic choices of density, and we therefore neglect it in our final calculations.

The validity of the average $T_{A}$ prescription of Eq. (32) is confirmed for light nuclei by observing that $U_{0}\left(r_{T}\right)$, Eq. (A.10), is preserved under this substitution up to second 


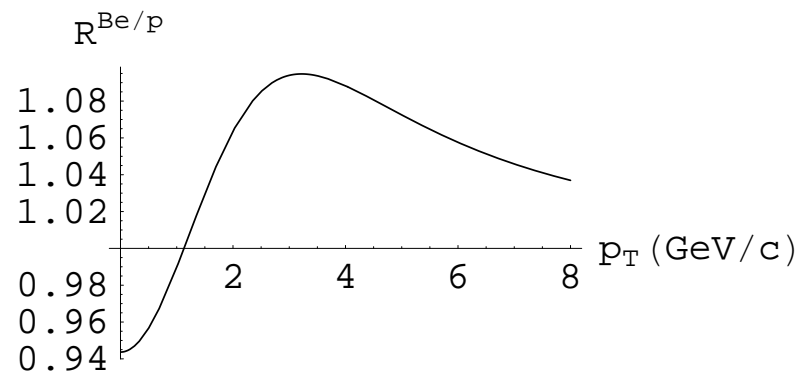

Figure 11: Nuclear ratio $R^{B e / p}\left(p_{T}\right)$ in the gaussian density model calculated using Eq. (A.13), including the center-of-mass correction.

order in $T_{A}$ in its multiple scattering expansion. This observation explains why the uniform density and the gaussian density models work essentially equally as well for ${ }^{9} B e$. It is perhaps surprising that the average thickness function prescription works so well even for heavy nuclei, when the higher order multiple scattering terms are important. The result is

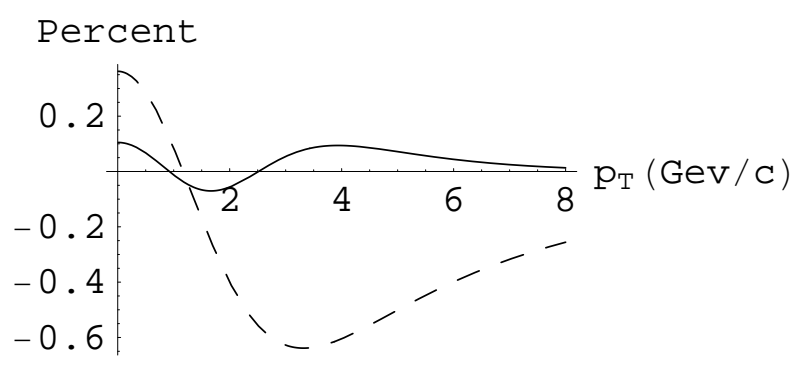

Figure 12: Comparison of $R_{G D}^{B e / p}$ and $R_{A T F}^{B e / p}$ (solid curve); relative contribution of center-of-mass correction to $R_{G D}^{B e / p}$ in the gaussian density model (dashed curve). See text for details. 
however intuitive, and it shows that on the average the quark propagates through half the nucleus before undergoing a Drell-Yan reaction. Based on our comparisons of the average thickness function approximation to numerical calculations in models, we conclude that the average thickness function approximation is justified for applications to data, and we will use it in the main body of the paper for calculating $\sigma_{D Y}^{p A}$. 


\section{References}

[1] G.T. Bodwin, S.J. Brodsky and G.P. Lepage, Phys. Rev. D 39, 3287 (1989).

[2] E772 Collaboration, D. M. Alde et al., Phys. Rev. Lett. 66 (1991) 133.

[3] D. M. Alde et al., Phys. Rev. Lett. 64 (1990) 2479.

[4] Unpublished E772 data, see in http://p25ext/lanl.gov/e866/e866.html

[5] M. A. Vasiliev, et al., Phys. Rev. Lett. 83 (1999) 2304.

[6] M. B. Johnson, et al., Phys. Rev. Lett. 86 (2001) 4483 (hep-ex/0010051); and M. B. Johnson, et al., Phys. Rev. C65 (2002) 025203 (hep-ph/0105195).

[7] P. L. McGaughey, J. M. Moss, and J.-Ch. Peng, NU-COLEX 99, Wako, Japan, 1999 (hep-ph/9905444); Annu. rev. Nucl. Part. Sci. 49 (1999) 217.

[8] M. B. Johnson, B. Z. Kopeliovich, M. J. Leitch, P. L. McGaughey, J. M. Moss, I. K. Potashnikova, I. Schmidt, hep-ph/0606126.

[9] M. Luo, J. Qiu, and G. Sterman, Phys. Rev. D50 (1994) 1951.

[10] X. Guo, J. Qiu, X. Zhang, Phys. Rev. Lett. 84 (2000) 5049.

[11] E. Wang and X.-N. Wang, Phys. Rev. Lett. 89 (2002) 162301.

[12] I. Vitev, Phys. Lett. B562 (2003) 36.

[13] R. Baier, Yu. L. Dokshitzer, A. H. Mueller, S. Peigne and D. Schiff, Nucl. Phs. B484 (1997) 265.

[14] J. Dolejsi, J. Hüfner and B. Z. Kopeliovich, Phys. Lett. B312 (1993) 235.

[15] M. B. Johnson, B. Z. Kopeliovich, and A. V. Tarasov, Phys. Rev. C63 (2001) 035203.

[16] A. Accardi and D. Treleani, Phys. Rev. D64 (2001) 116004.

[17] M. A. Betemps and M. B. Gay Ducati, Phys. Rev. D70 (2004) 116005; hep-ph/0505143; Phys. Lett. B636 (2006) 46.

[18] R. J. Glauber, High Energy Collision Theory in Lectures in Theoretical Physics, vol. 1, W. E. Brittin and L. G. Duham (eds.), Interscience, New York.

[19] V. N. Gribov, Sov. Phys. JETP 29 (1969) 483; ibid 30 (1970) 709; [Zh. Eksp. Teor. Fiz. 57 (1969) 1306].

[20] B. Z. Kopeliovich J. Raufeisen, and A. V. Tarasov, Phys. Lett. B 440, (1998) 151; J. Raufeisen, A. V. Tarasov, and O. O. Voskresenskaya, Eur. Phys. J. A5 (1999) 173. 
[21] J. Raufeisen, Phys. Lett. B557 (2003) 184.

[22] J. Webb, New Mexico State University Thesis (2002) (hep-ex/0301031).

[23] B.Z. Kopeliovich, L.I. Lapidus and A.B. Zamolodchikov, Sov. Phys. JETP Lett. 33 (1981) 612 .

[24] N. N. Nikolaev and B. G. Zakharov, Z. Phys. C49 (1991) 607.

[25] B. Z. Kopeliovich, Proc. of the Workshop Hirschegg '95: Dynamical Properties of Hadrons in Nuclear Matter, Hirschegg, January 16-21, 1995, ed. by H. Feldmeyer and W. Nörenberg, Darmstadt, 1995, p. 102 (hep-ph/9609385)

[26] S. J. Brodsky, A. Hebecker, and E. Quack, Phys. Rev. D55 (1997) 2584.

[27] K. Golec-Biernat and M. Wüsthoff, Phys. Rev. D59 (1998) 014017 (hep-ph/9807513); Phys. Rev. D60 (1999) 114023 (hep-ph/9903358).

[28] J. Raufeisen, J-C. Peng, and G. C. Nayak, Phys. Rev. D66 (2002), 034024 (hep-ph/0204095); B. Z. Kopeliovich, J. Raufeisen, and A. V. Tarasov, Phys. Lett. B503 (2001) 91; M. A. Betemps, M. B. Gay Ducati, M. V. T Machado, and J. Raufeisen, Phys. Rev.D 67 (2003) 114008.

[29] B. Z. Kopeliovich, J. Raufeisen, A. V. Tarasov, and M. B. Johnson, Phys. Rev. C67 (2003) 014903 (hep-ph/0105195).

[30] B.Z. Kopeliovich, A. Schäfer and A. V. Tarasov, Phys. Rev. C59 (1999) 1609 (hep-ph/9808378).

[31] Review of Particle Properties, Rev. Mod. Phys. 56 (1984).

[32] B. Z. Kopeliovich, J. Nemchik, I. K. Potashnikova, M. B. Johnson, and I. Schmidt, Phys. Rev. C72 (2005) 054606.

[33] B. Kopeliovich, J. Raufeisen, and A. Tarasov, Phys. Rev. C62 (2000) 035204.

[34] V. Karmanov and L. A. Kondratyuk, Sov. Phys. JETP Lett. 18 (1973) 266.

[35] P. McGaughey, private communication.

[36] M. Leitch, private communication.

[37] R. Rosenfelder, Phys. Lett. B 479 (2000) 381.

[38] See plots and tables on the E866 web page, http://p25ext/lanl.gov/e866/e866.html

[39] B. Kopeliovich, A. Schäfer, and A. Tarasov, Phys. Rev. D 62 (2000) 054022 (hep-ph/9908245). 
[40] I.K. Potashnikova, Private communication

[41] Atomic Data and Nuclear Data Tables, 36 (1987). 